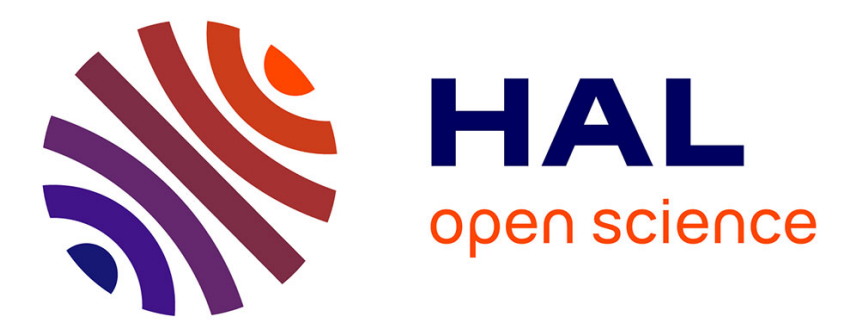

\title{
Feasible Speeds for Two Optimal Periodic Walking Gaits of a Planar Biped Robot
}

Mathieu Hobon, Víctor De-León-Gómez, Gabriel Abba, Yannick Aoustin, Christine Chevallereau

\section{- To cite this version:}

Mathieu Hobon, Víctor De-León-Gómez, Gabriel Abba, Yannick Aoustin, Christine Chevallereau. Feasible Speeds for Two Optimal Periodic Walking Gaits of a Planar Biped Robot. Robotica, inPress, 40 (11), pp.377-402. 10.1017/S0263574721000631 . hal-03242271

\section{HAL Id: hal-03242271 \\ https://hal.science/hal-03242271}

Submitted on 30 May 2021

HAL is a multi-disciplinary open access archive for the deposit and dissemination of scientific research documents, whether they are published or not. The documents may come from teaching and research institutions in France or abroad, or from public or private research centers.
L'archive ouverte pluridisciplinaire HAL, est destinée au dépôt et à la diffusion de documents scientifiques de niveau recherche, publiés ou non, émanant des établissements d'enseignement et de recherche français ou étrangers, des laboratoires publics ou privés. 


\title{
Feasible Speeds for Two Optimal Periodic Walking Gaits of a Planar Biped Robot
}

\author{
Mathieu Hobon†, Víctor De-León-Gómezł, Gabriel Abba†, \\ Yannick Aoustinł*, and Christine Chevallereauł
}

†4 rue Augustin Fresnel, 57078 Metz Cedex 3

Laboratoire de Conception Fabrication Commande, EA 4495

Arts et Métiers ParisTech, Université de Lorraine, France.

$\ddagger 1$, rue de la Nö̈, BP 92101. 44321 Nantes, France,

Laboratoire des Sciences du Numérique

de Nantes, UMR 6004,

CNRS, École Centrale de Nantes, Université de Nantes, France.

(Accepted MONTH DAY, YEAR. First published online: MONTH DAY, YEAR)

\section{SUMMARY}

The purpose is to define the range of feasible speeds for two walking motions for a particular planar biped robot, which differ in the definition of their finite time double support phases. For each speed, these two walking motions are numerically obtained by using a parametric optimization algorithm, regarding a sthenic criterion. Results allow us to define the range of allowable speeds for each walking. One result is that the first gait is less consuming in energy for moderate to fast velocity with respect to the second one, while the second gait is more efficient for low walking velocity.

KEYWORDS: Walking gait; Finite time double; Impact with foot flat; Toe impact; Heel impact.

\section{Introduction}

This paper explores the range of feasible speeds for a planar biped that adopts two walking motions, which differ in the definition of their finite time double support phases. The choice here of a planar biped is due to the fact that for human's walking gait the main movements are executed in the sagittal plane, see. ${ }^{1,2}$ Frontal plane movements mainly serve to keep human laterally balanced. The pelvis rotation has an important effect to limit the necessary energy to move the swing leg. ${ }^{3}$ However, our purpose being to study the energy effect of two finite time support phases, we assume that the results are little influenced by the pelvis rotation for the two gaits. The human walking is composed of single support phases and double support phases. For one step, the duration of a double support phase represents almost $24 \%$ of the time step. ${ }^{4}$ Several papers are devoted to a borderline case where the step of walking is composed of a single support phase and an instantaneous double support. ${ }^{5}$ For example the relatively human-inspired ones, which have been tested with the biped robots $A_{m b e r}{ }^{6}$ and Rabbit ${ }^{7}$ or the walking motions defined through the linear inverted pendulum ${ }^{8}$ or, ${ }^{9}$ among others.

However, for a biped robot, the contribution of the finite time double support phase is important to change the velocity rate of the walking, to increase the domain of stability in order to reject some disturbance occurring in single support, see, ${ }^{10}$ and $^{11}$ where planar

\footnotetext{
* Corresponding author. E-mail: Yannick.Aoustin@univ-nantes.fr
} 
bipeds with pointed feet are considered. Inserting a finite time double support phase in a gait of a biped with feet implies to deal with the behavior of the foot and the over actuation of the locomotor system, since a closed chain is formed by the locomotor system and the ground. There is a actuation redundancy leading to an infinite number of solutions for joint torques and the ground reaction wrench. Ju and Mansour, ${ }^{12}$ proposed a foot model with a curved planter surface to design a finite time double support phase for the motion of a biped in sagittal plane. Sharma and Stein, ${ }^{13}$ incorporate the finite time double support phases and single support phases in walking of a biped with point feet to minimize muscle activation and arm reaction forces generated from the walker. The dynamics-based optimization of sagittal gait cycles of a seven-link planar biped with feet by using the Pontryagin maximum principle is considered in. ${ }^{14}$ In there, one step is composed of a fully actuated single support phase and a finite time double support phase and the velocity of the swing foot at the landing on the ground is zero. Six periodic gaits are presented in. ${ }^{15}$ The simplest periodic gait is composed of successive single support phases with a flat foot contact on the ground, the stance foot does not rotate. The support phases are separated by instantaneous double support. The most complex periodic motion is composed of single support phases and finite time double support. For the finite time double support phases, the front foot rotates around its heel and the rear foot rotates around its toe. For this walking, with a finite time double support phase there is no impact of the swing foot landing on the ground, as well as for the walking gait defined in ${ }^{10}$ for a biped with point foot and in ${ }^{16}$ where an impactless walking gait is carried out with single support phases and finite time double support phases for a seven-link planar biped with feet. Nevertheless, it has been shown that the energy cost for the walking gait is smaller when the single support phases are ended with impacts than when the velocity of the swing foot at the landing is null. ${ }^{15}$ An original design is proposed for the knee joints of a planar biped robot, based on a four-bar linkage. A comparison of the performances with respect to a sthenic criterion is proposed between a biped equipped with four-bar knees and the other with revolute joints for walking reference trajectories composed of single phases, impact and finite time double support phases with rotation of both feet. ${ }^{17}$ The numerical results show that the performances with a four-bar linkage are worst for the smaller velocities and better for the higher velocities. Tan et al $l^{18}$ proposed a finite time double support phase that begins when the swing foot strikes the ground and finishes with the support foot toe-off. The inverse dynamic model is used to optimize a walking gait with the objective that the trunk remains upright. The criterion is based on the altitude of the center of mass with respect to a reference. Simulation results show that a two-level control strategy for simultaneous gait generation and stable control of planar walking of the ATRIAS biped can reject initial condition disturbances, while generating stable and steady walking motion. ${ }^{19}$

Despite all these interesting studies on walking motions with finite time double support phases, there is a lack of knowledge about the role of the feet during the finite time double support phase regarding on the speed of the biped robot. Several questions on this issue are still open, such as:

- Can one foot stay on the ground with a flat foot contact after its landing while the other rotates?

- Can both feet rotate simultaneously after the landing of the swing foot on its heel?

- What are the feasible speeds for walking with finite time double support phase? Feasible speed means here, speed that satisfies the limits of the actuator and the unilateral constraints of the biped robot with the ground.

- What is the best strategy at impact to satisfy the unilateral constraints on both feet for a feasible speed?

The goal of this paper is to see if we can draw on anthropomorphic features to improve the walking of bipedal robots with rigid feet. Of course to design a human-like walking there are many other interesting questions such as a foot-roll design, ${ }^{20}$ or the rotation of the foot during the single stance phase. ${ }^{15}$ But we want to focus our attention on the effect of finite time double support phases for walking. In particular the goal is to 
give a response to the four previous research questions for a set of periodic walking motions in the sagittal plane which are composed of impacts, single support phases and finite time double support phases. The ground and the biped limbs are assumed rigid. These gaits are defined with a parametric optimization by using a sthenic criterion and with nonlinear constraints. Even if for human there are movements of bodies that take place in the frontal plane, we limit our numerical studies in the sagittal plane because the magnitude of movements are much larger. As a consequence, the results about the energy consumption that are obtained in the sagittal plane are usually confirmed by a study in the $3 \mathrm{D}$ space, see for example. ${ }^{17}$ The planar biped model is defined with the physical parameters of the experimental biped Hydroid. ${ }^{21}$ Its inertial parameters are close to those of human. The step of the first gait is composed of a single support phase, with support on flat foot, a flat-footed impact on the ground for the swing leg, and a finite time double support phase, where the rear foot rotates around its toe and the front foot is kept flat on the ground. With this first walking motion it is possible to answer "Yes" to the first question. The second walking motion design is similar to the first one, except that the single support is ended by a heel impact and during the finite time double support phase the rear foot rotates on its toe and the forward foot rotates on its heel. The finite time double support of this gait is ended with a toe impact on the ground of the forward foot. With this second walking motion it is possible to answer "Yes" to the second question. According to the experimental studies of Winter ${ }^{4}$ this second walking motion is closer to the human walking motion than the first one. Studying these two walking motions, for various speed, will allow to answer the third and fourth questions. The algorithm for defining optimal walking gait has been carefully studied and programmed to converge to physically feasible solutions. When convergence was not achieved, it means that the bipedal robot could not achieve the walking gait with the target speed.

This paper is outlined as follows. Section 2 gathers the definition of the two walking motions. Section 3 presents the biped modeling for each phase of the walking motions. Section 4 deals with the trajectory planning. In section 5 , numerical results of the criterion evaluation as a function of the bipedal robot speed are presented. Finally, section 6 offers our conclusion and proposes several perspectives.

\section{Studied Gaits}

In the following, two types of walking motions are studied. A lot of articles are devoted to the definition of walking speeds with single support phases, instantaneous double supports $^{22}$ or with single support phases, finite time double support phases but without impact, ${ }^{16}$ In order to offer the reader a simple and rigorous presentation, two walking more anthropomorphic motions with phases of simple support and finite time double support and impact, will be compared.

- Gait 1: The periodic motion is composed of single support and finite time double support phases. At the end of the single support phase, see Fig. 1a, there is a flatfooted impact on the ground. In double support phase the rear foot (foot 2) rotates on its toe, the other is flat on the ground, see Fig. 1b. On Fig. 1c, the finite time double support is ended when the rear foot (foot 2) takes off the ground with the toe, the other foot stays flat on the ground. The single support takes place, Fig. 1d.

- Gait 2: The periodic motion is composed of single support and finite time double support phases. At the end of the single support phase, see Fig. 2a, the impacting foot (now foot 1) touches the ground with its heel. The rear foot (foot 2) keeps contact with the ground through its toe. In double support phase both feet rotate, see Fig. 2b. The finite time double support ends when the front foot (foot 1) impacts the ground with its toe and the rear foot (foot 2) takes off as shown in Fig. 2c. Then the single support takes place, see Fig. 2 d.

The gait 1 maximizes the walking stability since the support area in finite time double support is larger than the one of the gait 2. The gait 2 allows larger walking velocity 
a)

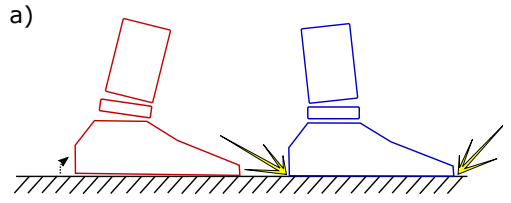

b)

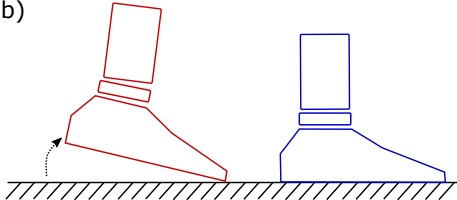

c)

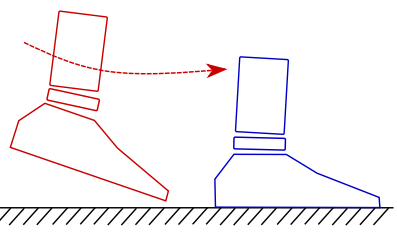

d)

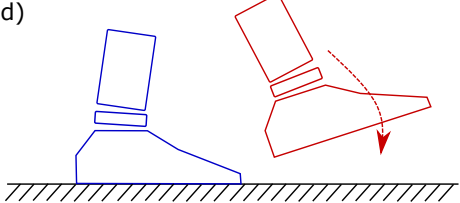

Fig. 1. Walking motion 1. a) End of the SS beginning of the DS b) DS c) End of the DS d) SS

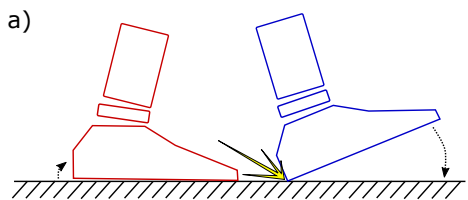

b)

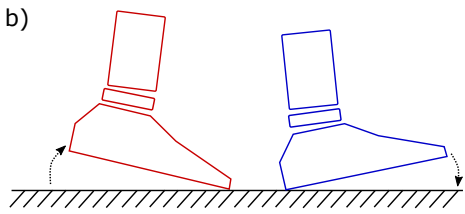

c)
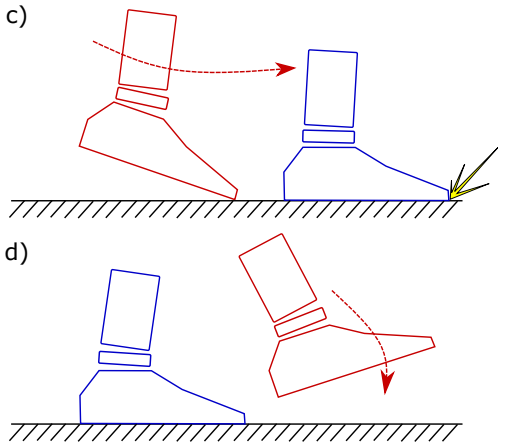

Fig. 2. Walking gait 2. a) End of the SS / beginning of the DS b) DS c) End of the DS d) SS

than gait 1 since the distance between foot can be increased while respecting the joint limits. 


\section{The biped modeling}

\subsection{The biped}

Let us consider a seven-link planar biped robot with physical parameters obtained from those of the $3 D$ experimental biped Hydroid ${ }^{21}$ and. ${ }^{23}$ A photography of the locomotor system of Hydroid is shown in Fig. 3. The inertial parameters of Hydroid are inspired from the Hanavan model. ${ }^{24}$ The considered planar biped is depicted in Fig. 4. Table I gathers its physical parameters. The parameters $s_{i}, i=1, \ldots, 5$ define the position of the center of mass of the limbs and the trunk with respect to the hip and knee joints. The parameters $l_{f}$ and $L_{f}$ are the distances from the projection of the joint ankle on the foot sole with the heel and the toe, respectively. $H_{f}$ is the distance between the ankle joint and the sole. $S_{p x}$ and $S_{p y}$ are the coordinates of the center of mass $G_{f}$ of the foot with respect to the ankle joint. The lengths of the shins, thighs, and the trunk are respectively $l_{1}, l_{2}$, and $l_{t}$.

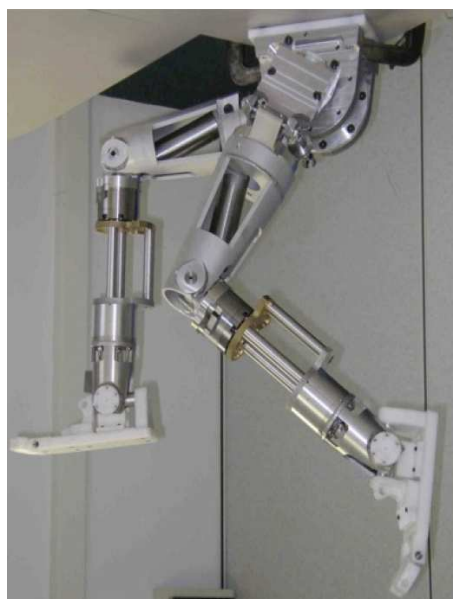

Fig. 3. Photography of the locomotor system of Hydroid.

\begin{tabular}{|c|c|c|c|c|}
\hline & Mass (kg) & Length (m) & $\begin{array}{c}\text { Moment of inertia } \\
\left(\mathrm{kg} . \mathrm{m}^{2}\right)\end{array}$ & $\begin{array}{c}\text { Center of } \\
\text { mass }(\mathrm{m})\end{array}$ \\
\hline foot & $m_{f}=0.7$ & $\begin{array}{c}L_{f}=0.21 \\
l_{f}=0.07 \\
H_{f}=0.07\end{array}$ & 0.002 & $s_{f x}=0.01$ \\
& & $s_{f y}=0.03$ & \\
\hline shin & 2.2 & $l_{1}=0.4$ & 0.03 & $s_{1}=s_{4}=0.17$ \\
\hline thigh & 5.0 & $l_{2}=0.4$ & 0.07 & $s_{2}=s_{3}=0.17$ \\
\hline trunk & 29.0 & $l_{t}=0.4$ & 0.8 & $s_{5}=0.2$ \\
\hline
\end{tabular}

Table I . Physical parameters of the biped robot..

\subsection{Dynamic modeling: General case}

Let us introduce the generalized vector $\mathbf{q}$ for the description of the considered biped as follows ${ }^{1}$ :

$$
\mathbf{q}=\left[q_{f_{1}}, q_{f_{2}}, q_{1}, q_{2}, q_{3}, q_{4}, q_{5}, x, y\right]^{\top} .
$$

The generalized variables $x$ and $y$ are the Cartesian coordinates of the hip joint. The other generalized variables are shown in Fig. 4. The vector $\mathbf{q}$ is chosen with nine components including $x$ and $y$ in order to be able to define a dynamic model that explicitly takes into account the unilateral constraints with the ground. For the studied walking motions the

\footnotetext{
${ }^{1}$ Sign $T$ means transposed vector or transposed matrix in this paper.
} 


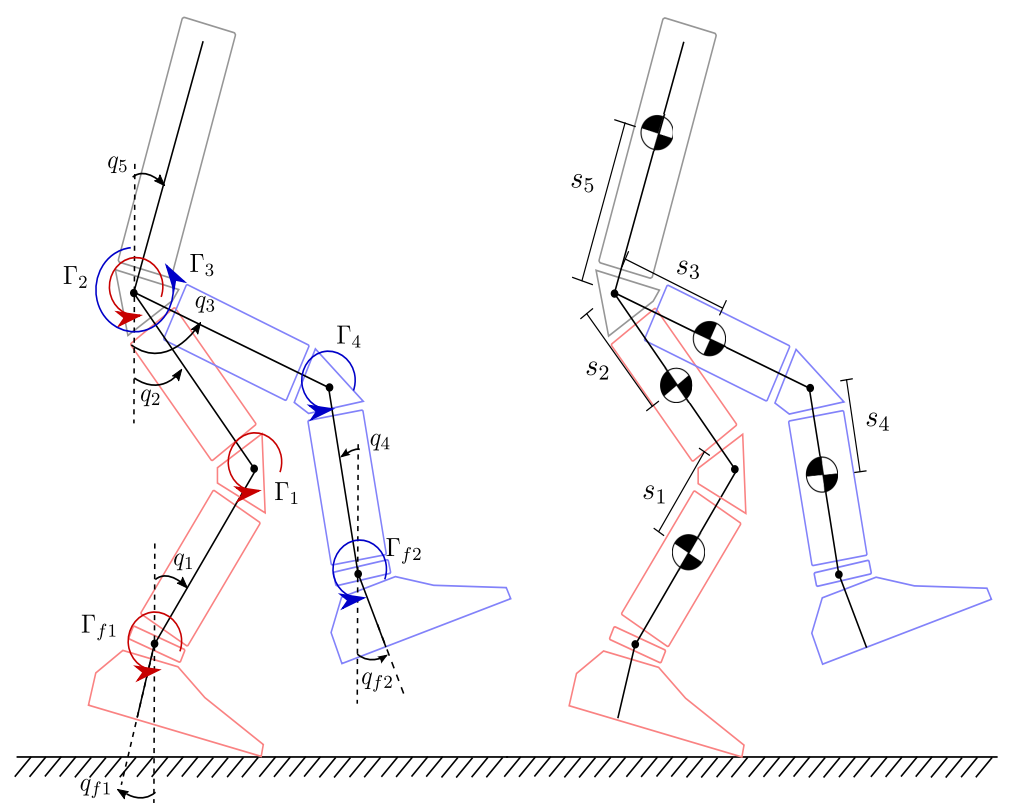

Fig. 4. Schematic of the planar biped robot. Absolute angular variables and joint torques (The angular variables are counted positive counterclockwise).

contact with the ground of the biped can be with the whole sole, the heel, or the toe, see Figs. 1 and 2.

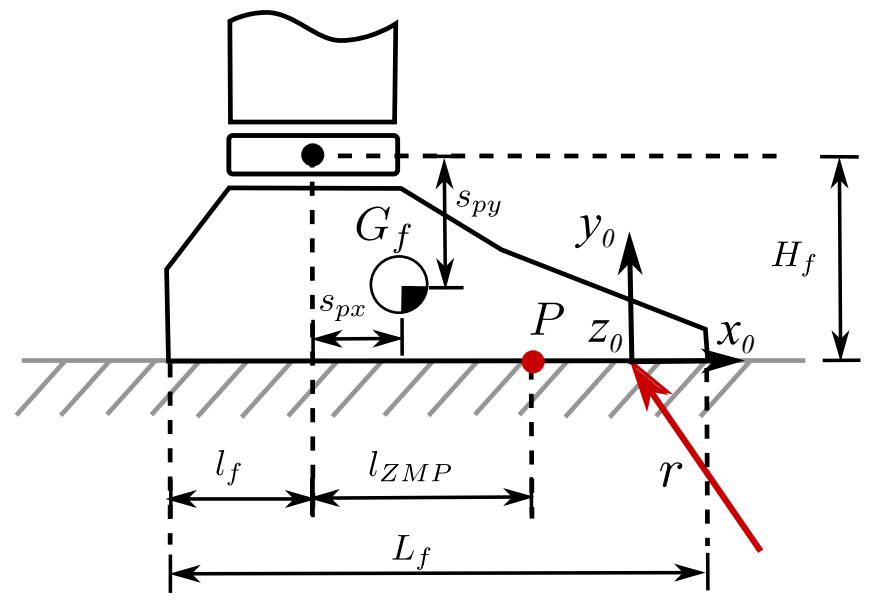

Fig. 5. Details of the foot.

For any type of contact of the feet with the ground, the dimension of the robot given in Figs. 4 and 5 allows to write the condition to ensure a rigid contact between the feet and the ground based on geometric relation. Here, no slipping of the stance foot is assumed. Thus, the hypothesis of rigid contact implies zero velocity and zero acceleration of the foot relative to the ground.

By considering the virtual work principle, the matrix $\mathbf{J}_{i}^{\top}$ that allows to take into account the ground reaction $\mathbf{r}_{i}=\left[r_{i x}, r_{i y}, \mathcal{M}_{i}\right]^{\top}$ in the dynamic model. This vector $\mathbf{r}_{i}$, defined in a frame $\left(x_{0}, y_{0}, z_{0}\right)$, see Fig. 5 , represents the wrench corresponding to the reaction force and moment from the ground acting on foot $i$. If only one punctual contact between foot $i$ and the ground is considered, the component of moment in $\mathbf{r}_{i}$ (for a frame attached to the foot) is zero, i.e. $\mathbf{r}_{i}=\left[r_{i x}, r_{i y}\right]^{\top}$. 
Thus, the dynamic model of the biped robot is expressed as:

$$
\mathbf{D}(\mathbf{q}) \ddot{\mathbf{q}}+\mathbf{N}(\mathbf{q}, \dot{\mathbf{q}})+\mathbf{Q}(\mathbf{q})=\mathbf{B} \boldsymbol{\Gamma}+\mathbf{J}_{1}^{\top}(\mathbf{q}) \mathbf{r}_{1}+\mathbf{J}_{2}^{\top}(\mathbf{q}) \mathbf{r}_{2},
$$

with the constraint equations

$$
\mathbf{J}_{i}(\mathbf{q}) \ddot{\mathbf{q}}+\dot{\mathbf{J}}_{i}(\mathbf{q}, \dot{\mathbf{q}}) \dot{\mathbf{q}}=0 \quad \text { for } \quad i=1 \text { to } 2 .
$$

Here, $\mathbf{D} \in \mathbb{R}^{9 \times 9}$ is the symmetric positive inertia matrix of the biped. Vector $\mathbf{N} \in \mathbb{R}^{9 \times 1}$ represents the centrifugal and Coriolis effects, and $\mathbf{Q} \in \mathbb{R}^{9 \times 1}$ is the effect of gravity vector. $\mathbf{B} \in \mathbb{R}^{9 \times 6}$ is a constant input mapping matrix composed of 1 and $0 . \boldsymbol{\Gamma} \in \mathbb{R}^{6 \times 1}$ is the vector of applied joint torques. $\mathbf{J}_{1}^{\top}$ and $\mathbf{J}_{2}^{\top}$ are the $9 \times 2$ (or $9 \times 3$ with a flat foot contact) transposed Jacobian matrices converting the ground reaction wrench at feet 1 and 2 into torques applied at joints by considering a rigid contact. For the rigid contact of the foot 1 with the ground, the corresponding equations, in position, velocity, and acceleration are introduced in Appendix A. In this Appendix equations (A2) and (A4) describe a contact with the heel, and the Jacobian matrix, denoted in this case $J_{1}=J_{h 1}$ is given in equation (A3). Equations (A6) and (A8) describe a contact with the toe, and the Jacobian matrix, denoted in this case $J_{1}=J_{t 1}$ is given in equation (A7). Equations (A10) and (A12) are written for a foot contact flat on the ground with no take-off and no slipping of the whole sole, and the Jacobian matrix denoted in this case $J_{1}=J_{f 1}$ is given in equation (A11).

Equations (1) and (2) allow to describe any contact of the feet with the ground. These equations are usual in literature, however the case of finite time double support is not often considered in detail. In this paper, an analysis of this phase is carried out in section 4.

\subsection{Impact model}

In biped walking, an impact usually occurs when the swing foot touches the ground. For gait 2, an impact may also occur when the rear leg toe touches the ground. Let $T$ be the instant of the impact. An absolutely inelastic impact is assumed, so that the foot does not slip. Given these conditions, the ground reactions at the instant of an impact can be considered as impulsive forces and defined by Dirac delta-functions. Impact equations can be obtained through the integration of the equation of motion (1) for the infinitesimal time from $T^{-}$to $T^{+}$. The vector of actuated torques and the Coriolis and gravity vectors have finite values. Thus, they do not affect the impact equations. Consequently the impact equations can be written in the following matrix form:

$$
\mathbf{D}(\mathbf{q}(T))\left(\dot{\mathbf{q}}^{+}-\dot{\mathbf{q}}^{-}\right)=\mathbf{J}_{I 1}^{\top}(\mathbf{q}(T)) \mathbf{i}_{1}+\mathbf{J}_{I 2}^{\top}(\mathbf{q}(T)) \mathbf{i}_{2} .
$$

Here, $\mathbf{q}(T)$ denotes the generalized coordinates of the biped at instant $t=T$, (these generalized coordinates does not change at the instant of the impact), $\dot{\mathbf{q}}^{-}$and $\dot{\mathbf{q}}^{+}$are respectively the velocity vectors just before and just after an inelastic impact. $\mathbf{J}_{I 1}$ and $\mathbf{J}_{I 2}$ characterize the contact of legs 1 and 2 with the ground during the impact, $\mathbf{i}_{1}$ and $\mathbf{i}_{2}$ are the wrenches corresponding to the impulsive forces and moments from the ground reaction acting on feet 1 and 2 respectively.

For the studied gaits a finite time double support phase is desired, thus for the first impact (Figs. 1a and 2b) one does not want the 1 foot to remain flat on the ground. Extensive simulations have shown that take-offs of the rear point foot can be avoided only if the landing velocity of the swing foot is zero ${ }^{10}$ and therefore, there is no impact either. This solution has a high torque cost. Thus, in the following it is assumed that only the toe of the rear foot remains on the ground after the impact. First let us consider the walking motion 1 . The swing foot hits the ground with a flat foot contact as shown 
in Fig. 1a. In this case, the impact model is as follows:

$$
\begin{gathered}
\mathbf{D}(\mathbf{q}(T))\left(\dot{\mathbf{q}}^{+}-\dot{\mathbf{q}}^{-}\right)=\mathbf{J}_{f 1}^{\top}(\mathbf{q}(T))\left[\begin{array}{c}
i_{1 x} \\
i_{1 y} \\
i_{\mathcal{M} 1}
\end{array}\right]+\mathbf{J}_{t 2}^{\top}(\mathbf{q}(T))\left[\begin{array}{c}
i_{2 x} \\
i_{2 y}
\end{array}\right] \\
{\left[\begin{array}{c}
\mathbf{J}_{f 1}(\mathbf{q}(T)) \\
\mathbf{J}_{t 2}(\mathbf{q}(T))
\end{array}\right] \dot{\mathbf{q}}^{+}=\mathbf{0}_{5 \times 1},}
\end{gathered}
$$

where the use of matrices $J_{f 1}$ and $J_{t 2}$ denotes that after impact the foot 1 has a foot flat contact with the ground and the foot 2 has a contact with the toe.

Let us consider now the walking motion 2. For one step, there are two impacts. The first impact occurs at the end of the single support phase when the swing foot heel impacts the ground as shown in Fig. 2a. This impact is described by the next equation:

$$
\begin{gathered}
\mathbf{D}(\mathbf{q}(T))\left(\dot{\mathbf{q}}^{+}-\dot{\mathbf{q}}^{-}\right)=\mathbf{J}_{h 1}^{\top}(\mathbf{q}(T))\left[\begin{array}{l}
i_{1 x} \\
i_{1 y}
\end{array}\right]+\mathbf{J}_{t 2}^{\top}(\mathbf{q}(T))\left[\begin{array}{l}
i_{2 x} \\
i_{2 y}
\end{array}\right] \\
{\left[\begin{array}{c}
\mathbf{J}_{h 1}(\mathbf{q}(T)) \\
\mathbf{J}_{t 2}(\mathbf{q}(T))
\end{array}\right] \dot{\mathbf{q}}^{+}=\mathbf{0}_{4 \times 1},}
\end{gathered}
$$

where the use of matrices $J_{h 1}$ and $J_{t 2}$ denotes that after impact the foot 1 has a heel contact with the ground and the foot 2 has a toe contact with the ground. During the double support phase the foot 2 rotates on its toe while the front foot rotates on its heel. The second impact occurs at the end of the double support phase, when the toe of the front foot reaches the ground and a flat-footed impact with the ground occurs while the rear foot takes off (see Fig. 2c). This second impact is described by the next equation:

$$
\begin{gathered}
\mathbf{D}(\mathbf{q}(T))\left(\dot{\mathbf{q}}^{+}-\dot{\mathbf{q}}^{-}\right)=\mathbf{J}_{f 1}^{\top}(\mathbf{q}(T))\left[\begin{array}{c}
i_{1 x} \\
i_{1 y} \\
i_{\mathcal{M} 1}
\end{array}\right] \\
\mathbf{J}_{f 1}(\mathbf{q}(T)) \dot{\mathbf{q}}^{+}=\mathbf{0}_{3 \times 1} .
\end{gathered}
$$

For each impact, when the velocity vector $\dot{\mathbf{q}}^{-}$just before the impact is known, the resolution of the systems (4), (5), or (6) gives the velocity vector $\dot{\mathbf{q}}^{+}$just after the impact and the impulsive reaction efforts $\mathbf{i}_{1}$ and $\mathbf{i}_{2}$.

\section{Gait optimization for the periodic walking}

In this section we present the algorithm for defining optimal walking trajectories. Each step of this algorithm has been carefully defined in order to minimize one sthenic criterion and to take into account all the physical and technological constraints that make walking possible. This is the core of our work.

To deal with a minimum energy walking, the Pontryagin's principle can be used. This principle is used by Rostami and Besonnet ${ }^{16}$ to design impactless walking motions for a seven-link planar biped robot with feet. However the calculations are complex, the resulting equations are highly sensitive to the initial conditions, and this method generates bang-bang control laws. ${ }^{25}$ Direct collocation method is an alternative to define walking motions. The principle of this method is to approach the solution of an ordinary differential equation or a partial differential equation for a finite set of points $,{ }^{26},{ }^{27},{ }^{28},{ }^{13},{ }^{18}$ and. ${ }^{29}$ The parametrization of the problem and the conversion of it into an algebraic optimization problem is another efficient alternative. Torques, Cartesian coordinates or joint coordinates can be chosen to define the optimization parameters. Discrete values for the torques are used as optimization variables in. ${ }^{30}$ However, a numerical integration 
of the direct dynamic model is necessary to find the reference trajectory in velocity and position. To overcome this difficulty authors in, ${ }^{31},{ }^{32}$ and ${ }^{33}$ propose respectively polynomial functions and truncated Fourier series to approximate the temporal evolution of the joints, then torques are found through the algebraic solution of the inverse dynamic model. Cartesian coordinates are also convenient as optimization parameters, ${ }^{34}$ but this choice requires the use of inverse geometric model and for a given posture of the biped singularities can appear.

In this paper a parametric optimization method is used and the evolution of a set of independent joint variables are expressed as polynomial functions of time. The coefficients of these polynomial functions define a set of desired initial, final, and intermediate positions and velocities. From the polynomial functions, we can calculate their first and second time derivatives. By using the inverse dynamic model, we can deduce the joint torques. A criterion, based on the joint torques is minimized to define cyclic walking motions by considering optimization variables among the set coefficients of the polynomial functions.

\subsection{Principle}

The generalized coordinates of the biped are given by vector $\mathbf{q}$. However these generalized coordinates are not independent due to the equation describing the contact with the ground. The locomotor system forms a geometrical closed loop with the ground. Thus it is not possible to choose all these generalized coordinates arbitrarily. Among the set of generalized coordinates several coordinates can be defined as function of time. The evolution of the other variables are then deduced based on geometrical relations that take into account the contact with the ground. The number of constraints varies with the phases of the motion (single support and finite time double support).

Between two successive phases, the position and velocity of the biped must be continuous or the discontinuity must satisfy the impact equations (4), (5), or (6). The set of parameters $P$ specifying the cubic spline functions are determined by taking into account the transition conditions between the following phases.

The motion studied is assumed to be periodic and with the same behavior on support on legs 1 and 2. This periodic motion is designed with only one step. As consequence the initial configuration at the beginning of the current step has to be deduced from the final configuration of the same step with a swapping role of the legs. Thus assuming that the leg 1 is the stance leg in single support, an exchange of the number of the joints is carried out using a matrix $\mathbf{A}$ as follows:

$$
\mathbf{q}^{+}=\mathbf{A q}^{-},
$$

where

$$
\mathbf{A}=\left[\begin{array}{lllllllll}
0 & 1 & 0 & 0 & 0 & 0 & 0 & 0 & 0 \\
1 & 0 & 0 & 0 & 0 & 0 & 0 & 0 & 0 \\
0 & 0 & 0 & 0 & 0 & 1 & 0 & 0 & 0 \\
0 & 0 & 0 & 0 & 1 & 0 & 0 & 0 & 0 \\
0 & 0 & 0 & 1 & 0 & 0 & 0 & 0 & 0 \\
0 & 0 & 1 & 0 & 0 & 0 & 0 & 0 & 0 \\
0 & 0 & 0 & 0 & 0 & 0 & 1 & 0 & 0 \\
0 & 0 & 0 & 0 & 0 & 0 & 0 & 1 & 0 \\
0 & 0 & 0 & 0 & 0 & 0 & 0 & 0 & 1
\end{array}\right],
$$

i.e. the initial configuration of the robot after the change of stance leg is the final configuration of the robot before the change of stance leg.

The set of optimization variables $P$ minimizing a sthenic criterion are searched using a non-linear optimization method. This algorithm is based on the calculation at each iteration of the gradient vector with respect to the optimization variables $P$, tacking into account of non-linear constraints. Physical conditions such as conditions of no slipping 
of the stance feet on the ground, of no unexpected contact with the ground of the transfer leg, and physical limits on the actuators define the non-linear constraints in this optimization process.

4.1.1. The criterion. A lot of criteria can be used to produce an optimal trajectory. The cost transport (COT) to evaluate the biped gaits is a common and good option. ${ }^{35}$ However to deal with a smoother mathematical function the sthenic criterion based on the squared torques is chosen to obtain optimal trajectories. As for a motor its maximum delivered torque is strongly connected to its weight the physical meaning of this sthenic criterion is also interesting:

$$
C_{W}=\frac{1}{d}\left(\int_{0}^{T} \boldsymbol{\Gamma}^{\top} \boldsymbol{\Gamma} \mathrm{d} t\right)=\frac{1}{d}\left(\int_{0}^{T_{S S}} \boldsymbol{\Gamma}^{\top} \boldsymbol{\Gamma} \mathrm{d} t+\int_{T_{S S}}^{T_{S S}+T_{D S}} \boldsymbol{\Gamma}^{\top} \boldsymbol{\Gamma} \mathrm{d} t\right)
$$

where $T, T_{S S}$, and $T_{D S}$ are respectively the durations of the step, the single support phase, and the finite time double support phase. Several motions with different velocities will be defined. When the walking speed $v$ is fixed, the step length $d$ and $T_{D S}$ are optimization variables. The step duration $T$ is directly given through the relation $T=d / v$. Thus we can deduce $T_{S S}$ as follows:

$$
T_{S S}=\frac{d}{v}-T_{D S}
$$

4.1.2. Parametric functions: Cubic spline. Cubic spline functions ${ }^{36}$ are used to define the trajectories $\theta_{i}(t)$ of each independent angular variable during a phase of the walking motion,

$$
\theta_{i}(t)=\left\{\begin{array}{c}
\varphi_{i, 1}(t) \text { if } t_{1} \leq t \leq t_{2} \\
\varphi_{i, 2}(t) \text { if } t_{2} \leq t \leq t_{3} \\
\cdot \\
\cdot \\
\cdot \\
\varphi_{i, n-1}(t) \text { if } t_{n-1} \leq t \leq t_{n} .
\end{array} \quad i=1,2, \ldots, n_{j}\right.
$$

Here, $n$ is the number of selected knots and $n_{j}$ is the number of angular variables. $\varphi_{i, 1}(t), \ldots, \varphi_{i, n-1}(t)$ are polynomials of third-order such that:

$$
\varphi_{i, k}(t)=\sum_{j=0}^{3} a_{i, k}^{j}\left(t-t_{k}\right)^{j}, \text { for } t \in\left[t_{k}, t_{k+1}\right], k=1, \ldots, n-1
$$

where the coefficients $a_{i, k}^{j}$ are calculated such that the trajectory, velocity, and acceleration are continuous between $t_{1}$ and $t_{n}$. The cubic spline functions are uniquely defined by specifying an initial angular position $\theta_{i}(0)$, an initial angular velocity $\dot{\theta}_{i}(0)$ (both at $t=t_{1}=0$ ), a final angular position $\theta_{i}(T)$, and a final velocity $\dot{\theta}_{i}(T)$ (both at $t=t_{n}=T$ ), with $T$ being the duration of the phase and $n-2$ intermediate angular positions $(n-2$ because let us recall that in finite time double support and single support phases the two Cartesian positions can be deduced from the knowledge of the angular positions). Consequently, the temporal joint evolution will be defined by a limited number of optimization parameters $\left((n+2) \times n_{j}\right)$. When functions $\theta_{i}(t)$ are chosen, the joint velocities and accelerations can be deduced through time derivation of the polynomial function $\theta_{i}(t)$.

The number of parameters increases with the number of knots $n$ but the order of the polynomial functions, the cubic spline functions does not increase. These cubic polynomials are sufficient to ensure the continuity of the second derivatives at nodes. 
4.2. Description of the gait in the different phases

4.2.1. The single support phase. The biped has a foot contact flat on the ground, see Fig. 1d or Fig. 2d. It means there are three unilateral constraints of contact in the stance foot with the ground. Thus there are only $\mathbf{9}-\mathbf{3}=\mathbf{6}$ independent generalized variables among the set of components of vector q. Let us assume without loss of generality that the biped is in single support on foot 1 . Then $q_{f_{1}}, \dot{q}_{f_{1}}$, and $\ddot{q}_{f_{1}}$ are null. We can choose as independent coordinates: $\Theta=\left[\theta_{1}, \theta_{2}, \theta_{3}, \theta_{4}, \theta_{5}, \theta_{6}\right]^{\top}=\left[q_{f_{2}}, q_{1}, q_{2}, q_{3}, q_{4}, q_{5}\right]^{\top}$. The duration of the single support phase is $T_{S S}=T-T_{D S}$. The cubic spline functions $\theta_{i}(t), i=1, \ldots, 6$ are defined with three selected knots, $n=3$ for this phase. Thus, for each joint we need to define five parameters of position and velocity to design the trajectories.

If the single support phase is ended by a flat-footed impact of the swing leg, only four independent variables are necessary to define the final configuration of the robot. For the choice of these four independent variables we use the distance $d$ between the front heel and the rear toe, see Fig. 6 , the position coordinates of the hip $x, y$, and the inclination of the torso $q_{5}$. If the single phase is ended by a heel impact of the swing leg five independent generalized coordinates are necessary to define the final configuration of the robot. The angle of the front foot has to be added. The final velocity of the joints at the single support phase is described with six variables for both cases.

4.2.2. The finite time double support phases. For gait 1, leg 1 has a flat foot contact and foot 2 rotates on its toe, as shown in Fig. 1a. Then, there are five unilateral constraints of contact with the ground. There are nine generalized variables and five constraint equations. Thus during the double support phase the biped's configuration can be described with only four independent coordinates. Let us choose the orientation $q_{f_{2}}$ of foot 2 , the orientation angles of leg $1 q_{1}, q_{2}$, and the inclination angle of the torso $q_{5}: \Theta=\left[\theta_{1}, \theta_{2}, \theta_{3}, \theta_{4}\right]^{\top}=\left[q_{f_{2}}, q_{1}, q_{2}, q_{5}\right]^{\top}$.

For gait 2, leg 1 rotates around its heel and foot 2 rotates on its toe, as shown in Fig. 2a. Then there are four unilateral constraints of contact with the ground. There are nine generalized variables and four constraint equations. Thus during the double support phase the biped's configuration can be described with only five independent coordinates. Let us choose: $\Theta=\left[\theta_{1}, \theta_{2}, \theta_{3}, \theta_{4}, \theta_{5}\right]^{\top}=\left[q_{f_{1}}, q_{f_{2}}, q_{1}, q_{2}, q_{5}\right]^{\top}$.

Let $T_{D S}$ be the duration of the double support phase. Only limited evolution of the joints exists during the double support phase. Thus the cubic spline functions $\theta_{i}(t)$, are defined with two selected knots for gait 1 and three for gait 2 . We can remark that the distance between feet $d$ is constant during this phase, thus the number of independent parameters to describe the robot configuration is reduced as it was shown.

4.2.3. Continuity of the generalized coordinates between phases. The studied gaits are periodic, and the different phases are connected via impact model with a jump of velocities or continuity between the generalized coordinates, thus the initial configuration and velocity of each phase can be deduced based on the final configuration and velocity of the previous phase. The number of optimization variables can thus be reduced and are summarized in the tables II and III. For the periodic walking motions 1 and 2, the number of knots for the single and double supports phases have been chosen in order to have similar number of optimization variables. For gait 1 , we choose $n_{S S}=4$ and $n_{D S}=2$ that gives 31 optimization variables, and for gait 2 , we choose $n_{S S}=3$ and $n_{D S}=3$ that gives 32 optimization variables. $T_{D S}$ and $d$ are also optimization variables. Tacking into account equation (9), the duration of the single support phase $T_{S S}$ can be deduced.

\subsection{The optimal torque}

When the motion of the biped is defined with the cubic functions as function of time (4.1.2), their first, and their second time derivatives can be calculated. The contact equation allows to define the vector $\mathbf{q}$ of generalized coordinates and its derivatives $\dot{\mathbf{q}}$ and $\ddot{\mathbf{q}}$. Then the dynamic model (1) can be used to deduce the torque and the criterion can be evaluated. In the case of double support phase, due to actuation redundancy, many torques produce the same motion. A local optimal problem can be stated to choose the specific torques in double support as a function of the ground reaction. To define this 


\begin{tabular}{|c|c|c|}
\hline Description & Optimization variables & $\begin{array}{l}\text { Number of } \\
\text { parameters }\end{array}$ \\
\hline $\begin{array}{l}\text { Final configuration } \\
\text { of single support }\end{array}$ & $x_{h}\left(T_{S S}\right), y_{h}\left(T_{S S}\right), q_{5}\left(T_{S S}\right)$ & 3 \\
\hline $\begin{array}{c}\text { Final configuration } \\
\text { of double support }\end{array}$ & $q_{1}\left(T_{D S}\right), q_{2}\left(T_{D S}\right), q_{5}\left(T_{D S}\right), q_{f 2}\left(T_{D S}\right)$ & 4 \\
\hline $\begin{array}{c}\text { Intermediate } \\
\text { configuration } \\
\text { of single support }\end{array}$ & $\begin{array}{c}q_{f 2}\left(k \frac{T_{S S}}{n_{S S}-1}\right), q_{1}\left(k \frac{T_{S S}}{n_{S S}-1}\right), q_{2}\left(k \frac{T_{S S}}{n_{S S}-1}\right), q_{3}\left(k \frac{T_{S S}}{n_{S S}-1}\right), \\
q_{4}\left(k \frac{T_{S S}}{n_{S S}-1}\right), q_{5}\left(k \frac{T_{S S}}{n_{S S}-1}\right), \mathrm{k}=1, \ldots, n_{S S^{-}}\end{array}$ & $6\left(n_{S S^{-}} 2\right)$ \\
\hline $\begin{array}{c}\text { Intermediate } \\
\text { configuration } \\
\text { of double support }\end{array}$ & $\begin{array}{c}q_{f 2}\left(k \frac{T_{D S}}{n_{D S}-1}\right), q_{1}\left(k \frac{T_{D S}}{n_{D S}-1}\right), q_{2}\left(k \frac{T_{D S}}{n_{D S}-1}\right), q_{5}\left(k \frac{T_{D S}}{n_{D S}-1}\right) \\
\mathrm{k}=1, \ldots, n_{D S}-2\end{array}$ & $4\left(n_{D S^{-}}-2\right)$ \\
\hline $\begin{array}{l}\text { Final velocities } \\
\text { of single support }\end{array}$ & $\dot{q}_{f 2}\left(T_{S S}\right), \dot{q}_{1}\left(T_{S S}\right), \dot{q}_{2}\left(T_{S S}\right), \dot{q}_{3}\left(T_{S S}\right), \dot{q}_{4}\left(T_{S S}\right), \dot{q}_{5}\left(T_{S S}\right)$ & 6 \\
\hline $\begin{array}{l}\text { Final velocities } \\
\text { of double support }\end{array}$ & $\dot{q}_{f 2}\left(T_{D S}\right), \dot{q}_{1}\left(T_{D S}\right), \dot{q}_{2}\left(T_{D S}\right), \dot{q}_{5}\left(T_{D S}\right)$ & 4 \\
\hline Step length & $d$ & 1 \\
\hline $\begin{array}{c}\text { Duration } \\
\text { of double support }\end{array}$ & $T_{D S}$ & 1 \\
\hline & $\overline{\text { Total }}$ & $\overline{\imath_{D S}+6 n_{S S}}$ \\
\hline
\end{tabular}

Table II . Number of optimization variables for the walking gait 1.

calculation we develop an explicit relation between the torque vector $\boldsymbol{\Gamma}$ and the wrench vector $\mathbf{r}_{2}$. Then we detail both cases, double support phases and single support phases and show the associated constraints.

4.3.1. Explicit relation between the torque vector $\boldsymbol{\Gamma}$ and the ground reaction in foot 2 $\mathbf{r}_{2}$. In finite time double support phase the locomotion system of the biped moves as a closed kinematic loop. This locomotion system is over-actuated. This situation requires an optimization process in order to manage the actuation redundancy and to find a solution that minimizes the criterion (8). This optimization process is based on an explicit relation between the torque vector $\boldsymbol{\Gamma}$ and the effort vector $\mathbf{r}_{2}$. From the dynamic model (1) we can write both following equations:

$$
\mathbf{B}^{\perp}(\mathbf{D} \ddot{\mathbf{q}}+\mathbf{N}+\mathbf{Q})=\mathbf{B}^{\perp}\left(\mathbf{J}_{1}^{\top} \mathbf{r}_{1}+\mathbf{J}_{2}^{\top} \mathbf{r}_{2}\right)
$$

and

$$
\mathbf{B}^{+}(\mathbf{D} \ddot{\mathbf{q}}+\mathbf{N}+\mathbf{Q})=\mathbf{\Gamma}+\mathbf{B}^{+}\left(\mathbf{J}_{1}^{\top} \mathbf{r}_{1}+\mathbf{J}_{2}^{\top} \mathbf{r}_{2}\right)
$$

Here, $\mathbf{B}^{\perp}(3 \times 9)$ and $\mathbf{B}^{+}(6 \times 9)$ are the orthogonal complement matrix and the pseudoinverse matrix of $\mathbf{B}$, respectively, i.e., $\mathbf{B}^{\perp} \mathbf{B}=\mathbf{0}_{3 \times 6}, \mathbf{B}^{+} \mathbf{B}=\mathbf{I}_{6 \times 6}$.

These equations (11) and (12) will be used in the next subsection to manage the over actuation of the biped in finite time double support and thus to manage an optimization of the torques in finite time double support phase. 


\begin{tabular}{|c|c|c|}
\hline Description & Optimization variables & $\begin{array}{c}\text { Number of } \\
\text { parameters }\end{array}$ \\
\hline \hline $\begin{array}{c}\text { Final configuration } \\
\text { of single support }\end{array}$ & $x_{h}\left(T_{S S}\right), y_{h}\left(T_{S S}\right), q_{5}\left(T_{S S}\right), q_{f 2}\left(T_{S S}\right)$ & 4 \\
\hline $\begin{array}{c}\text { Final configuration } \\
\text { of double support }\end{array}$ & $q_{1}\left(T_{D S}\right), q_{2}\left(T_{D S}\right), q_{5}\left(T_{D S}\right), q_{f 2}\left(T_{D S}\right)$ & 4 \\
\hline $\begin{array}{c}\text { Intermediate } \\
\text { configuration } \\
\text { of single support }\end{array}$ & $q_{f 2}\left(k \frac{T_{S S}}{n_{S S}-1}\right), q_{1}\left(k \frac{T_{S S}}{n_{S S}-1}\right), q_{2}\left(k \frac{T_{S S}}{n_{S S}-1}\right), q_{3}\left(k \frac{T_{S S}}{n_{S S}-1}\right)$, & $6\left(n_{S S}-2\right)$ \\
\hline $\begin{array}{c}\text { Intermediate } \\
\text { configuration } \\
\text { of double support }\end{array}$ & $q_{f 1}\left(k \frac{T_{D S}}{n_{D S}-1}\right), q_{f 2}\left(k \frac{T_{D S}}{n_{D S}-1}\right), q_{1}\left(k \frac{T_{D S}}{n_{D S}-1}\right), q_{2}\left(k \frac{T_{D S}}{n_{D S}-1}\right)$, & $5\left(n_{D S}-2\right)$ \\
\hline $\begin{array}{c}\text { Final velocities } \\
\text { of single support }\end{array}$ & $q_{f 2}\left(T_{S S S}\right), \dot{q}_{1}\left(T_{S S}\right), \dot{q}_{2}\left(T_{S S}\right), \dot{q}_{3}\left(T_{S S}\right), \dot{q}_{4}\left(T_{S S}\right), \dot{q}_{5}\left(T_{S S}\right)$ & 6 \\
\hline $\begin{array}{c}\text { Final velocities } \\
\text { of double support }\end{array}$ & $\dot{q}_{f 1}\left(T_{D S}\right), \dot{q}_{f 2}\left(T_{D S}\right), \dot{q}_{1}\left(T_{D S}\right), \dot{q}_{2}\left(T_{D S}\right), \dot{q}_{5}\left(T_{D S}\right)$ & \\
\hline Step length & $d$ & 5 \\
\hline $\begin{array}{c}\text { Duration } \\
\text { of double support }\end{array}$ & $T_{D S}$ & 1 \\
\hline \hline
\end{tabular}

Table III . Number of optimization variables for the walking gait 2 .

4.3.2. Optimal torques during the finite time double support phase for gait 1. Let us first consider the gait 1, through the resultant wrench of the ground reaction, which is composed of two components for each force applied on both feet and one component for the moment vector on the flat foot. The front leg has a flat foot contact (see Fig. 6a), thus the resultant wrench reaction of the ground acting in some point of this front foot is defined by $\mathbf{r}_{1}=\left[\begin{array}{lll}r_{1 x} & r_{1 y} & \mathcal{M}_{z}\end{array}\right]^{\top}$.

Let us consider the global equilibrium in translation and rotation of the biped, see Fig. 6a. Let $\delta_{g}$ be the dynamic momentum of the biped with respect to its center of mass defined by the Cartesian coordinates $\left(x_{g}, y_{g}\right)$. We have five unknown variables, $\mathcal{M}_{z}, r_{1 x}$, $r_{2 x}, r_{1 y}$, and $r_{2 y}$ for three equations only :

$$
\left\{\begin{array}{c}
y_{g}\left(r_{1 x}+r_{2 x}\right)+\left(x_{g}-d\right) r_{2 y}+\left(x_{g}+l\right) r_{1 y}+\mathcal{M}_{z}=\delta_{g} \\
r_{1 x}+r_{2 x}=m \ddot{x}_{g} \\
r_{1 y}+r_{2 y}-m g=m \ddot{y}_{g} .
\end{array}\right.
$$

Consequently, among $r_{1 x}, r_{2 x}, r_{1 y}, r_{2 y}$, and $\mathcal{M}_{z}$ two variables can be chosen as optimization variables. For a given sum $r_{1 x}+r_{2 x}$, there are an infinity of solutions for $r_{1 y}, r_{2 y}$, and $\mathcal{M}_{z}$ that satisfy the first and third equations of (13). Let $r_{2 x}$ and $r_{2 y}$ be the optimization variables. From (11) $\mathbf{r}_{1}$ is such as:

$$
\mathbf{r}_{1}=\left(\mathbf{B}^{\perp} \mathbf{J}_{1}^{\top}\right)^{-1} \mathbf{B}^{\perp}\left(\mathbf{D} \ddot{\mathbf{q}}+\mathbf{N}+\mathbf{Q}-\mathbf{J}_{2}^{\top} \mathbf{r}_{2}\right)
$$


assuming that $\mathbf{B}^{\perp} \mathbf{J}_{1}^{\top}$ is invertible as it has been tested in all our numerical tests.

Substituting $\mathbf{r}_{1}$ with its expression (14) in (12) the torque vector is such as:

$$
\begin{aligned}
\boldsymbol{\Gamma}= & \mathbf{B}^{+}\left(\mathbf{I}_{9 \times 9}-\mathbf{J}_{1}^{\top}\left(\mathbf{B}^{\perp} \mathbf{J}_{1}^{\top}\right)^{-1} \mathbf{B}^{\perp}\right)(\mathbf{D} \ddot{\mathbf{q}}+\mathbf{N}+\mathbf{Q})- \\
& \mathbf{B}^{+}\left(\mathbf{I}_{9 \times 9}-\mathbf{J}_{1}^{\top}\left(\mathbf{B}^{\perp} \mathbf{J}_{1}^{\top}\right)^{-1} \mathbf{B}^{\perp}\right) \mathbf{J}_{2}^{\top} \mathbf{r}_{2} .
\end{aligned}
$$

From equation (15) let us identify a linear relation, that emphasizes the effect of the optimization variable $\mathbf{r}_{2}$ on the actuated torque $\boldsymbol{\Gamma}$ :

$$
\boldsymbol{\Gamma}=\mathbf{M}-\mathbf{K r}_{2},
$$

Here, the size of the matrices $\mathbf{M}, \mathbf{K}$ is respectively $(6 \times 1)$, and $(6 \times 2)$ and:

$$
\begin{aligned}
& \mathbf{M}=\mathbf{B}^{+}\left(\mathbf{I}_{9 \times 9}-\mathbf{J}_{1}^{\top}\left(\mathbf{B}^{\perp} \mathbf{J}_{1}^{\top}\right)^{-1} \mathbf{B}^{\perp}\right)(\mathbf{D} \ddot{\mathbf{q}}+\mathbf{N}+\mathbf{Q}), \\
& \mathbf{K}=\mathbf{B}^{+}\left(\mathbf{I}_{9 \times 9}-\mathbf{J}_{1}^{\top}\left(\mathbf{B}^{\perp} \mathbf{J}_{1}^{\top}\right)^{-1} \mathbf{B}^{\perp}\right) \mathbf{J}_{2}^{\top} .
\end{aligned}
$$

For a given motion we can locally choose the solution that minimizes the criterion (8),

$$
\min _{r_{2 x}, r_{2 y}} \boldsymbol{\Gamma}^{\top} \boldsymbol{\Gamma} .
$$

By using the relation (16) the expression of $\boldsymbol{\Gamma}^{\top} \boldsymbol{\Gamma}$ can be written as

$$
\begin{aligned}
\boldsymbol{\Gamma}^{\top} \boldsymbol{\Gamma} & =\left(\mathbf{M}-\mathbf{K} \mathbf{r}_{\mathbf{2}}\right)^{\top}\left(\mathbf{M}-\mathbf{K r}_{\mathbf{2}}\right) \\
& =\mathbf{M}^{\top} \mathbf{M}-2 \mathbf{r}_{2}^{\top} \mathbf{K}^{\top} \mathbf{M}+\mathbf{r}_{2}^{\top} \mathbf{K}^{\top} \mathbf{K} \mathbf{r}_{2} .
\end{aligned}
$$

We have numerically checked that matrix $\mathbf{K}^{\top} \mathbf{K}$ is definite positive, thus the criterion $\boldsymbol{\Gamma}^{\top} \boldsymbol{\Gamma}$ as function of vector $\mathbf{r}_{2}$ is strictly convex and has a minimum. The solution $\mathbf{r}_{2}$ opt which minimizes $\boldsymbol{\Gamma}^{\top} \boldsymbol{\Gamma}$ can be calculated by writing that the derivative of $\boldsymbol{\Gamma}^{\top} \boldsymbol{\Gamma}$ with respect to $\mathbf{r}_{2}$ is equal to zero.

$$
\begin{aligned}
\frac{\partial}{\partial \mathbf{r}_{2}}\left(\boldsymbol{\Gamma}^{\top} \boldsymbol{\Gamma}\right)=0 \Rightarrow \mathbf{r}_{2} \text { opt } & =\left(\mathbf{K}^{\top} \mathbf{K}\right)^{-1} \mathbf{K}^{\top} \mathbf{M} \\
& =\mathbf{K}^{+} \mathbf{M} .
\end{aligned}
$$

The solution $\mathbf{r}_{2 \text { opt }}=\left[r_{2 x \text { opt }} r_{2 y \text { opt }}\right]^{\top}$ found with (19), minimizes $\boldsymbol{\Gamma}^{\top} \boldsymbol{\Gamma}$ without constraints. Remark: For $\mathbf{r}_{2}$ opt the constraints of no take-off and no slipping in double support can be satisfied or not. At this stage of the optimization algorithm we can search a solution $\mathbf{r}_{2}$ to satisfy the defined constraints. But another way, that we choose, is to reject the obtained global solution with the global optimization and the $S Q P$ optimization algorithm when a constraints is not satisfied.

4.3.3. Optimal torques during the double support phase for gait 2. For gait 2 the problem is similar but presents some differences. Let us consider the global equilibrium in translation and rotation of the biped (20), with Fig. 6b. The resultant reaction of the ground acting in the pivot point that represents the heel of the front foot 1 is defined by $\mathbf{r}_{1}=\left[\begin{array}{ll}r_{1 x} & r_{1 y}\end{array}\right]^{\top}$. Force $\mathbf{r}_{2}=\left[\begin{array}{ll}r_{2 x} & r_{2 y}\end{array}\right]^{\top}$ is the ground reaction acting in the toe of the rear foot $2, \delta_{g}$ is the dynamic momentum of the biped with respect to its center of mass. We have four unknown variables, $r_{1 x}, r_{2 x}, r_{1 y}$, and $r_{2 y}$ for three equations only.

$$
\left\{\begin{aligned}
y_{g}\left(r_{1 x}+r_{2 x}\right)+\left(x_{g}-d\right) r_{2 y}+x_{g} r_{1 y} & =\delta_{g} \\
r_{1 x}+r_{2 x} & =m \ddot{x}_{g} \\
r_{1 y}+r_{2 y}-m g & =m \ddot{y}_{g} .
\end{aligned}\right.
$$

For a given reference trajectory of the center of mass defined along with $x_{g}, \dot{x}_{g}$ and $\ddot{x}_{g}, m \ddot{x}_{g}$ is known, and then the sum $r_{1 x}+r_{2 x}$ through the second equation of (20). 

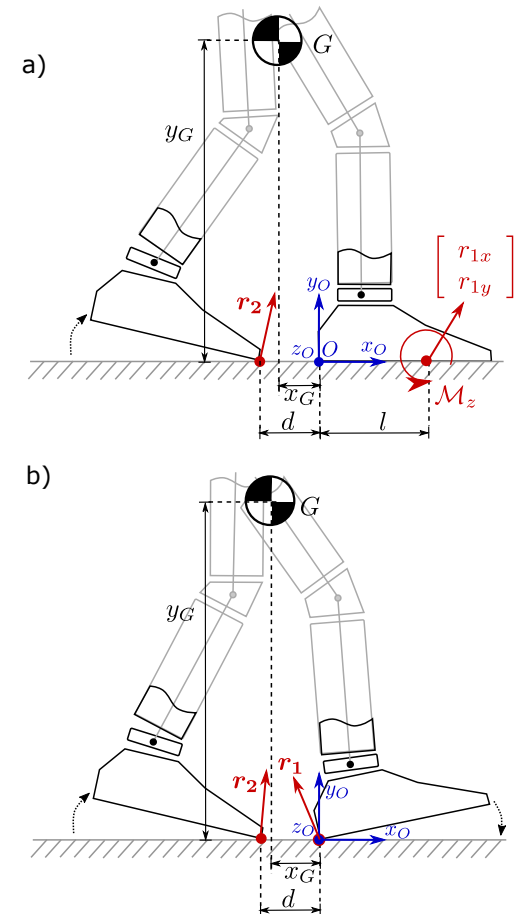

Fig. 6. Ground reactions in double support phase and the center of mass of the biped. a) DS of gait 1 b) DS of gait 2

Consequently $r_{1 y}$ and $r_{2 y}$ are the unique solution of the first and second equations of (20). But there are an infinity of solutions for $r_{1 x}$ or $r_{2 x}$ that satisfy the first and second equations of (20). Let $r_{2 x}$ be defined as an optimization variable to minimize locally the criterion (8). Using (11) we can write:

$$
\begin{aligned}
& \mathbf{B}^{\perp}(\mathbf{D} \ddot{\mathbf{q}}+\mathbf{N}+\mathbf{Q}) \\
& =\mathbf{B}^{\perp}\left(\mathbf{J}_{1}^{\top}\left[\begin{array}{l}
r_{1 x} \\
r_{1 y}
\end{array}\right]+\mathbf{J}_{2}^{\top}\left[\begin{array}{l}
r_{2 x} \\
r_{2 y}
\end{array}\right]\right), \\
& =\mathbf{B}^{\perp}\left(\mathbf{J}_{1}^{\top}\left[\begin{array}{c}
r_{1 x} \\
r_{1 y}
\end{array}\right]+\mathbf{J}_{21}^{\top} r_{2 x}+\mathbf{J}_{22}^{\top} r_{2 y}\right), \\
& =\mathbf{B}^{\perp}\left(\mathbf{J}_{1}^{\prime \top}\left[\begin{array}{c}
\mathbf{r}_{1} \\
r_{2 y}
\end{array}\right]+\mathbf{J}_{21} r_{2 x}\right),
\end{aligned}
$$

with $\mathbf{J}_{1}^{\prime \top}=\left[\begin{array}{ll}\mathbf{J}_{1}^{\top} \mathbf{J}_{22}^{\top}\end{array}\right]$ and $\mathbf{r}_{1}=\left[\begin{array}{ll}r_{1 x} & r_{2 x}\end{array}\right]^{\top}$.

Assuming that $\mathbf{B}^{\perp} \mathbf{J}_{1}^{\prime \top}$ is invertible (we observed that $\mathbf{B}^{\perp} \mathbf{J}_{1}^{\prime} \uparrow$ also is numerically invertible) we have:

$$
\left[\begin{array}{c}
\mathbf{r}_{1} \\
r_{2 y}
\end{array}\right]=\left(\mathbf{B}^{\perp} \mathbf{J}_{1}^{\prime}\right)^{-1} \mathbf{B}^{\top}\left(\mathbf{D} \ddot{\mathbf{q}}+\mathbf{N}+\mathbf{Q}-\mathbf{J}_{21}^{\top} r_{2 x}\right)
$$

From (12) we also can write:

$$
\mathbf{B}^{+}(\mathbf{D} \ddot{\mathbf{q}}+\mathbf{N}+\mathbf{Q})=\mathbf{\Gamma}+\mathbf{B}^{+}\left(\mathbf{J}_{1}^{\prime \top}\left[\begin{array}{c}
\mathbf{r}_{1} \\
r_{2 y}
\end{array}\right]+\mathbf{J}_{21} r_{2 x}\right)
$$


Combining (22) with (23) we obtain:

$$
\begin{aligned}
\boldsymbol{\Gamma}= & \mathbf{B}^{+}\left(\mathbf{I}_{9 \times 9}-\mathbf{J}_{1}^{\prime \top}\left(\mathbf{B}^{\perp} \mathbf{J}_{1}^{\prime \top}\right)^{-1} \mathbf{B}^{\perp}\right)(\mathbf{D} \ddot{\mathbf{q}}+\mathbf{N}+\mathbf{Q}) \\
& -\mathbf{B}^{+}\left(\mathbf{I}_{9 \times 9}-\mathbf{J}_{1}^{\prime \top}\left(\mathbf{B}^{\perp} \mathbf{J}_{1}^{\prime \top}\right)^{-1} \mathbf{B}^{\perp}\right) \mathbf{J}_{21}^{\top} r_{2 x} .
\end{aligned}
$$

From equation (24) let us identify the linear form in $r_{2 x}$ for $\boldsymbol{\Gamma}$ :

$$
\boldsymbol{\Gamma}=\mathbf{M}_{1}-\mathbf{K}_{\mathbf{1}} r_{2 x} .
$$

Here, the size of the matrices $\mathbf{M}_{1}, \mathbf{K}_{1}$ is $(6 \times 1)$ and:

$$
\begin{aligned}
& \mathbf{M}_{1}=\mathbf{B}^{+}\left(\mathbf{I}_{9 \times 9}-\mathbf{J}_{1}^{\prime}\left(\mathbf{B}^{\perp} \mathbf{J}_{1}^{\prime \top}\right)^{-1} \mathbf{B}^{\perp}\right)(\mathbf{D} \ddot{\mathbf{q}}+\mathbf{N}+\mathbf{Q}), \\
& \mathbf{K}_{1}=\mathbf{B}^{+}\left(\mathbf{I}_{9 \times 9}-\mathbf{J}_{1}^{\prime \top}\left(\mathbf{B}^{\perp} \mathbf{J}_{1}^{\prime}\right)^{-1} \mathbf{B}^{\perp}\right) \mathbf{J}_{21}^{\top} .
\end{aligned}
$$

By using the same methodology that for the gait 1 with similar expressions to (18) and (19) the optimal solution is :

$$
\begin{aligned}
\frac{\partial}{\partial r_{2 x}}\left(\boldsymbol{\Gamma}^{\top} \boldsymbol{\Gamma}\right) & =0 \\
\Rightarrow \quad r_{2 x ~ o p t} & =\mathbf{K}_{\mathbf{1}}^{+} \mathbf{M}_{\mathbf{1}} .
\end{aligned}
$$

\section{Remarks:}

- As the scalar term $\mathbf{K}_{1}^{\top} \mathbf{K}_{1}$ is strictly positive, $\boldsymbol{\Gamma}^{\top} \boldsymbol{\Gamma}$ as function of $r_{2 x}$ has a minimum.

- Similarly to gait 1 if $\mathbf{r}_{2 x \text { opt }}$ does not satisfy the constraints of no take-off and no slipping, the $S Q P$ optimization algorithm will reject the obtained solution in the global optimization.

\subsection{Parametric optimization problem}

By parameterizing the joint motion in terms of cubic spline functions, the optimization problem is reduced to a constrained parametric optimization problem of the form:

$$
\begin{array}{ll}
\text { Minimize } & C_{W}(\mathbf{P}) \\
\text { subject to } & \mathbf{g}_{j}(\mathbf{P}) \leq 0 \text { for } j=1,2, \cdots, l
\end{array}
$$

where $\mathbf{P}$ is the set of optimization variables. $C_{W}(\mathbf{P})$, which is the sthenic criterion (8), is minimized with $l$ inequality constraints $\mathbf{g}_{j}(\mathbf{P}) \leq 0$ to satisfy. The vector $\mathbf{g}_{j}(\mathbf{P}) \leq 0$ regroups the unilateral constraints of contact with ground reactions, the geometrical constraints and motor limits. The criterion and these constraints are given in the following sections.

4.4.1. The single support phase. The biped has a flat foot contact on the ground, Fig. 1c. The resultant wrench of the ground reaction is composed of two components for the force and one component for the moment. In the second foot the resultant wrench of the ground reaction is null (assuming that the stance foot is foot $1, r_{2}=0$ ).

The dynamic model (1) becomes:

$$
\mathbf{D} \ddot{\mathbf{q}}+\mathbf{N}+\mathbf{Q}=\mathbf{B} \boldsymbol{\Gamma}+\mathbf{J}_{1}^{\top} \mathbf{r}_{1}
$$

By knowing $\mathbf{q}, \dot{\mathbf{q}}, \ddot{\mathbf{q}}$, which satisfy (2), this matrix equation has a unique solution for the torque vector $\boldsymbol{\Gamma}$ and the ground reaction effort $\mathbf{r}_{1}$.

4.4.2. The constraints. Two types of constraints are used to obtain a realistic gait.

- The contact constraints, which ensure a valid walking.

The first constraint ensures the stance leg does not take off or slide on the ground. The vertical component of the ground reaction of the foot must be positive. Furthermore, 
the ground reaction force is inside a friction cone, defined with the coefficient of friction $\mu_{f}$ :

$$
\left\{\begin{array}{l}
-r_{j y}<0 \\
\left(-\mu_{f} r_{j y}-r_{j x}\right) \leq 0 \\
\left(-\mu_{f} r_{j y}+r_{j x}\right) \leq 0
\end{array}\right.
$$

$j=1$ and/or 2. $r_{j x}$ and $r_{j y}$ are the normal and tangential components of the reaction force. Moreover, we can introduce a constraint on the ground reaction at the impact:

$$
\left\{\begin{array}{l}
-i_{j y}<0 \\
\left(-\mu_{f} i_{j y}-i_{j x}\right) \leq 0 \\
\left(-\mu_{f} i_{j y}+i_{j x}\right) \leq 0
\end{array}\right.
$$

$j=1$ and/or 2 . We choose an arbitrary numerical value for the friction coefficient $\mu_{f}$ equals to 0.7 . To ensure the non-rotation of the stance flat foot we introduce a constraint on the $Z M P$ during contact phase and at the instant of the impact:

$$
-l_{f} \leq l_{Z M P} \leq L_{f}-l_{f} .
$$

$l_{Z M P}$ represents the distance between the $Z M P$ and the projection of the ankle joint on the ground, see Fig. 5.

Just after the impact, the velocity of the taking-off foot should be directed upward. As a consequence, the positivity of the vertical component of the velocities for the heel and the toes is added to the set of constraints.

The next constraint allows to ensure the non penetration of the swinging foot in the ground. Defining the altitude of the toe and the heel of the foot 2 from expressions in Appendix (6) we obtain inequality constraints as follows

$$
\begin{gathered}
y-l_{2} \cos q_{3}-l_{1} \cos q_{4}+\left(L_{f}-l_{f}\right) \sin q_{f 2}-H_{f} \cos q_{f 2} \geqslant 0, \\
\quad \text { and } \\
y-l_{2} \cos q_{3}-l_{1} \cos q_{4}-l_{f} \sin q_{f 2}-H_{f} \cos q_{f 2} \geqslant 0 .
\end{gathered}
$$

- The geometrical constraints and actuator limits to ensure a technological realistic gait:

For the joint variables of the knee we limit the domain of desired solutions such as the knee counterflexion is avoided. Moreover, Table IV gathers the motor limits in torque, velocity, and power for the joints of the hips, knees and the ankles. These maximum values, are those of the locomotor system of the hydroid robot, which the power supply fluid is hydraulic. They are used for the two gaits and for each velocity of the optimal walking.

\begin{tabular}{|c|c|c|c|}
\hline & Hip joint & Knee joint & Ankle joint \\
\hline Maximum torque (N.m) & 30 & 10 & 20 \\
\hline Maximum velocity (rad/s) & 6 & 10 & 20 \\
\hline Maximum power (W) & 180 & 100 & 300 \\
\hline
\end{tabular}

Table IV . Actuator limits in torques and velocities..

A block diagram that summarize the parametric optimization algorithm proposed in this paper to define the two gaits is described in Fig 7.

\section{Optimal walking: Simulation results}

The problem of parametric minimization with constraints to obtain the optimal walking is numerically solved, using the $S Q P$ method see ${ }^{37}$ and $^{38}$ with the fmincon function of 


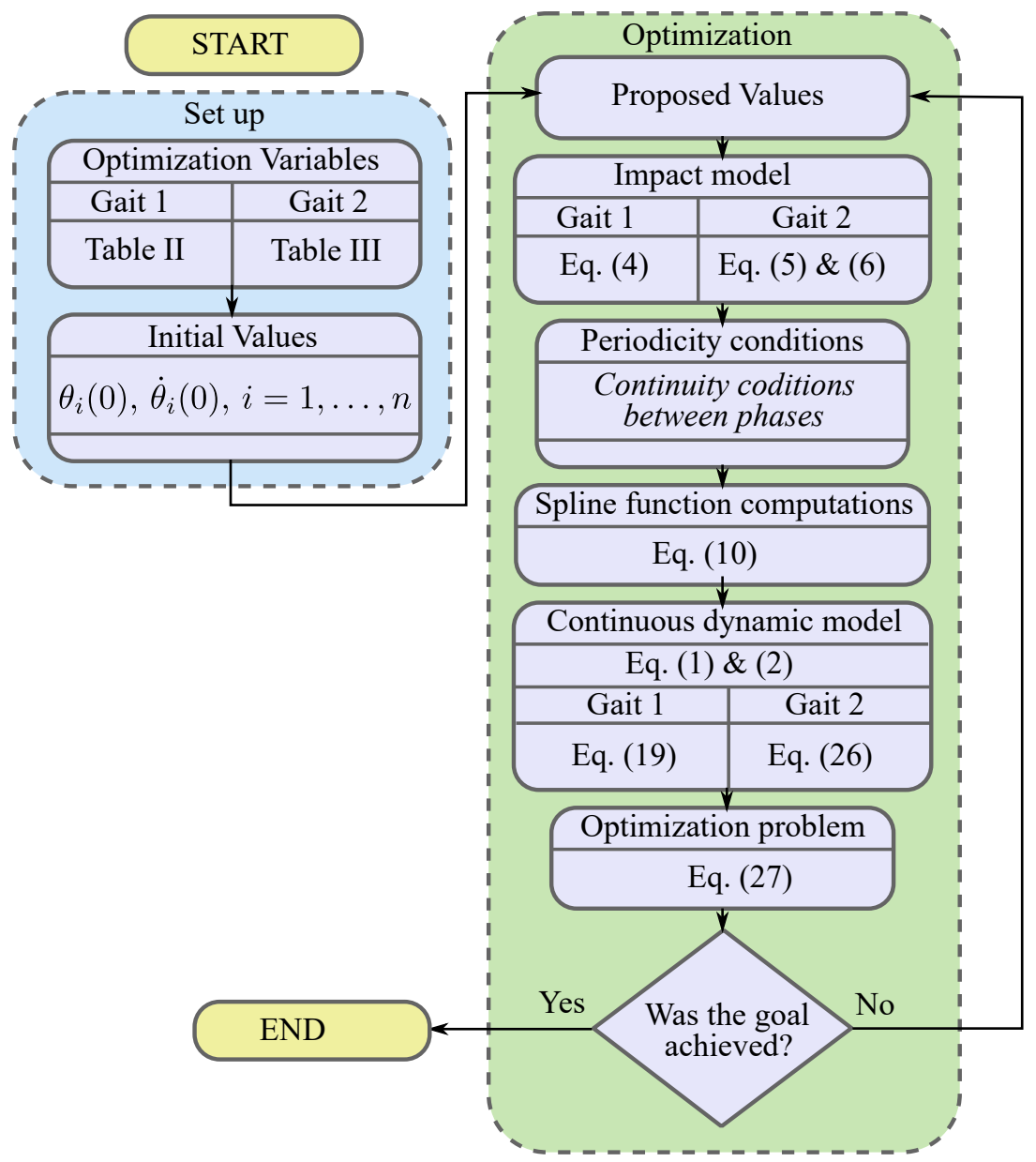

Fig. 7. Block diagram of the parametric optimization for defining the two gaits.

Matlab $\cap$. Figure 8 shows the cost criterion as function of the walking velocity of the biped for both gaits. The choice of the initial conditions for the optimization process is very important. The described curves are the results of several iterative optimization tests, by adapting the initial conditions for the current velocity from the previous velocity, starting from the lower or the higher allowable speed. For the gait 1, a cyclic motion has been found for walking velocity between $0.22 \mathrm{~m} / \mathrm{s}$ and $1 \mathrm{~m} / \mathrm{s}$. The criterion has a minimum around $0.36 \mathrm{~m} / \mathrm{s}$. The energy increases quasi linearly for higher walking velocities. For gait 2 , the optimization algorithm does not converge outside the velocity interval $[0.5 \mathrm{~m} / \mathrm{s}, 0.94 \mathrm{~m} / \mathrm{s}]$. However, walking velocities faster than $0.55 \mathrm{~m} / \mathrm{s}$ have values of the cost criterion lower than those obtained with the gait 1 . In the sense of speed walking, a synchronized rotation of both feet during the double support phase is more efficient than a flat foot contact on ground and a rotation around the toe of the other foot. We observed with numerical results that for both gaits with the impact model (3) at the end of the single support the velocity of the landing foot is small. This is in agreement with the numerical analyze made by Miossec and Aoustin. ${ }^{39}$ The reason is that, it is very difficult to include a finite time double support after a discontinuity of the velocity of the landing foot and simultaneously to satisfy the unilateral constraints of friction and no take-off with an absolutely inelastic impact model. 


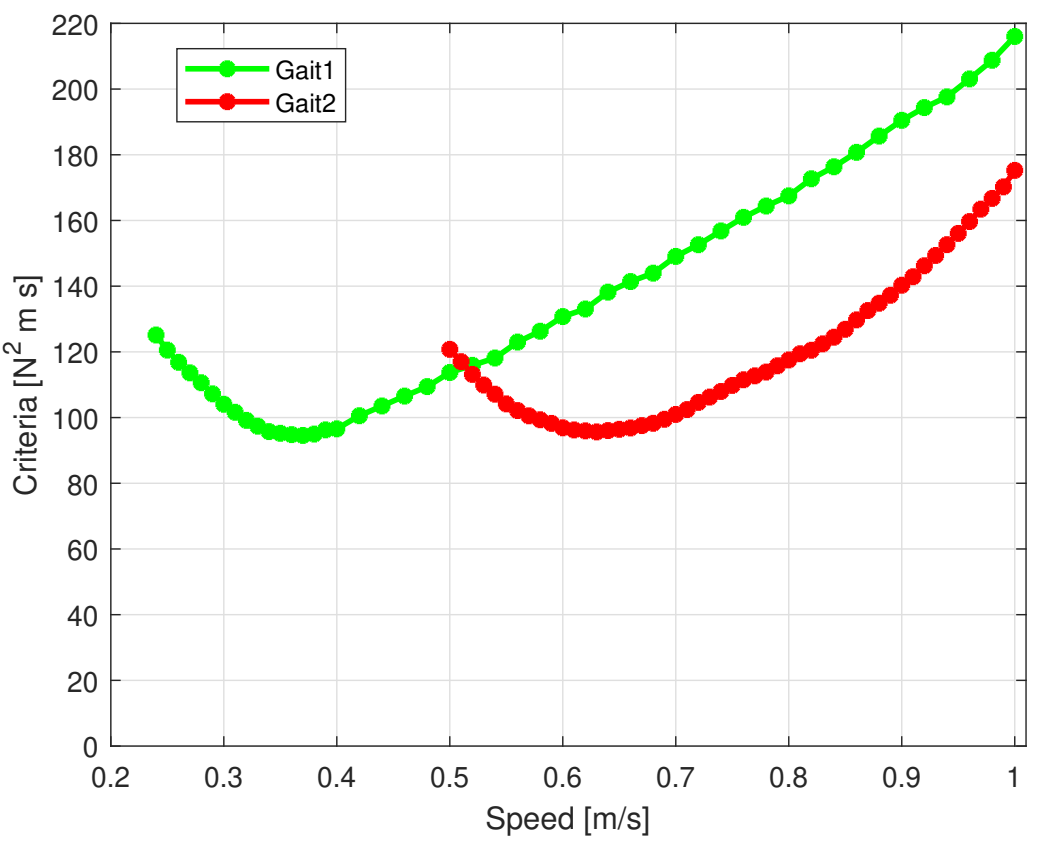

Fig. 8. Cost criterion as function of the walking velocity for gait 1 (green) and gait 2 (red).

Fig. 8, shows the ranges of feasible speeds for both gaits 1 and 2. Outside the speed ranges, the actuators can no longer provide enough power to perform the operation or the optimization algorithm cannot find a solution, which satisfies the unilateral constraints of the biped robot with the ground. The speed range is larger for the gait 1 than for the gait 2. However, for the common domain of both gaits, the sthenic criterion is weaker for the 2 gait, especially around the comfort speed of a healthy adult human, that is almost equal to $4.4 \mathrm{~km} / \mathrm{h} \pm 0.8,{ }^{40}$ This fact leads us to believe that gait 2 is more anthropomorphic than gait 1. Parametric optimization by definition provides a minimal solution of a criterion that is not an optimal solution in the Pontryagin sense. In reality for each speed, with another criterion, another strategy for choosing the initial value of the optimized variables, different written of the constraints, it may be possible to find a different robot motion. However, the multiple numerical tests that led us to these results, proved that the following general trend is true regardless of the trajectory calculation method: Gait 1 is better at low speed and worse at high speed than gait 2. From Fig. 8 we choose the velocity $0.9 \mathrm{~m} / \mathrm{s}(3.24 \mathrm{~km} / \mathrm{h})$ to detail the walking for both gaits. This is not far from the comfort velocity. Figs 9 and 10 describe a stick diagram for one step of the walking motion with gaits 1 and 2 respectively. 


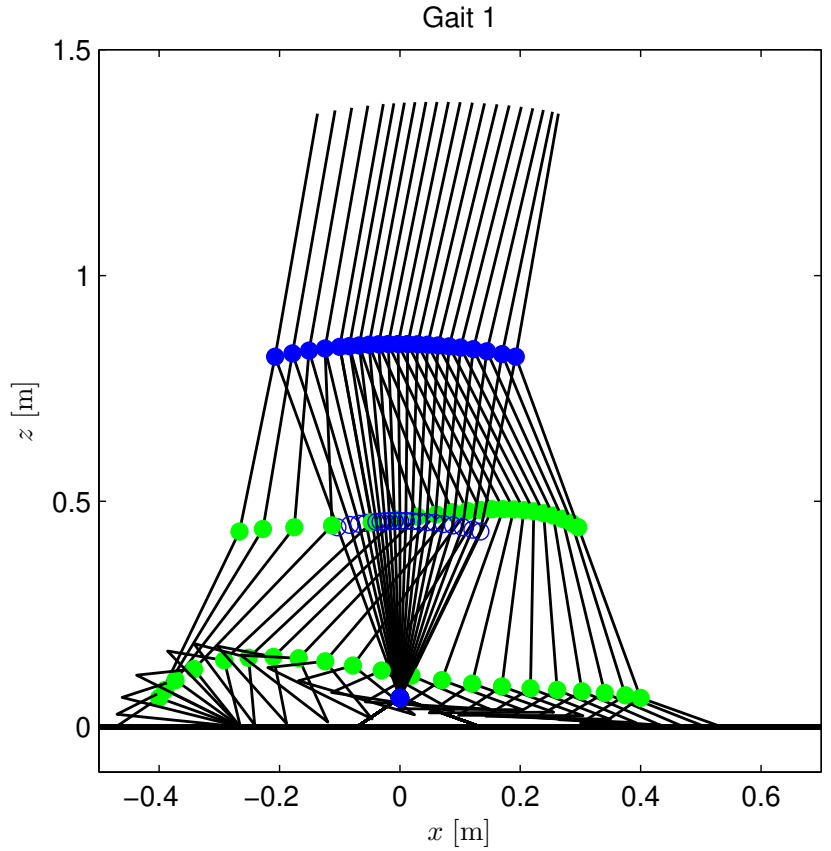

Fig. 9. Stick diagram of the gait 1.

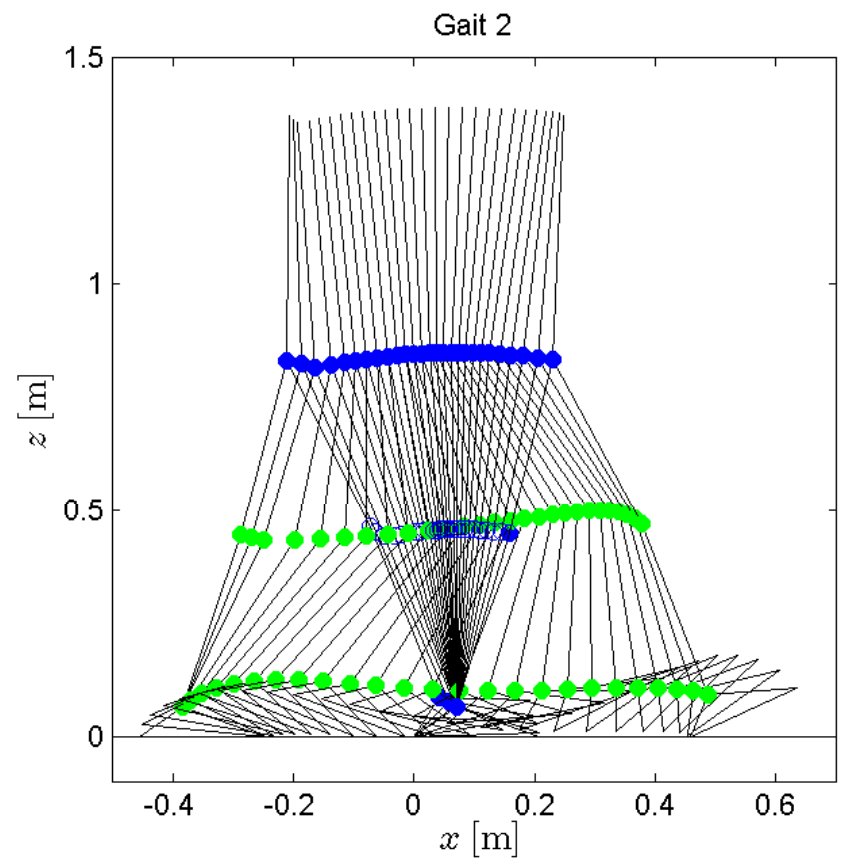

Fig. 10. Stick diagram of the gait 2 . 


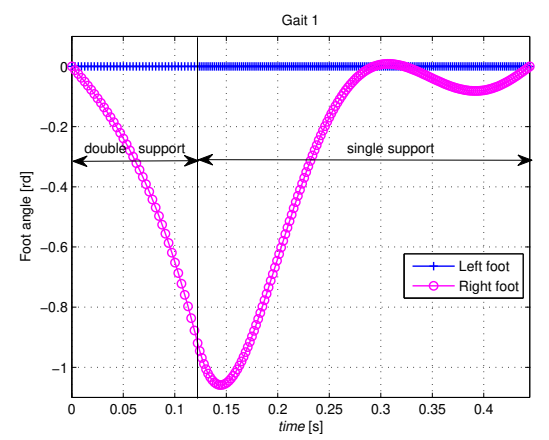

Fig. 11. Profile of the foot orientations with respect to the ground for the gait 1.

Figure 11 presents the orientation variables of the feet for gait 1 . We can observe the flat foot contact of the stance foot on the ground. The value of the orientation of its sole is null with respect to the ground. Figure 12 shows the profile of the torques and, the discontinuities of the torques, allows us to discern the impact at $t=0$, and the transition between the double support phase and the single support phase, which occurs at $0.12 \mathrm{~s}$. The profile of the torques show a discontinuity at the impact $(\mathrm{t}=0)$ because there is a jump of velocities, that is coherent with the definition of the gait 1 . During the double support phase, the rear foot rotates around its toe until $0.12 s$ and after it becomes the swing foot.

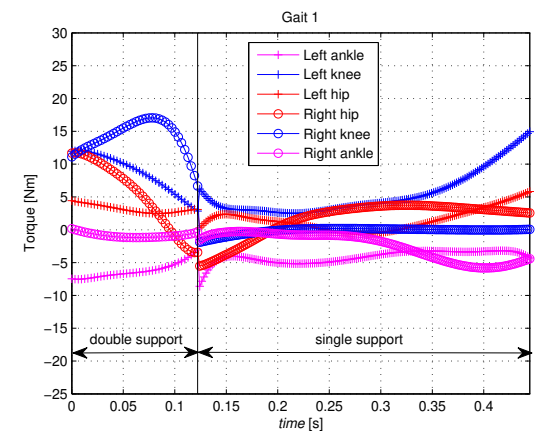

Fig. 12. Profile of the torques for the gait 1.

Figure 13 presents the orientation variables of the feet for gait 2. We can explicitly see the end of the finite time double support phase. The front foot rotates around its heel until $0.04 s$ when the orientation of its sole is null. The orientation value of the rear foot is almost zero during the double support phase, however the numerical results prove that there is a small rotation of this foot around its toe. After the impact of the front feet, the rear foot becomes the swing foot and its rotation increases. Figure 14 shows the profile of the torques and, at the discontinuities of the torques, the transition between phases. Similarly to gait 1 the profile of the torques show a discontinuity at the impacts because there is a jump of velocities, that is also coherent with the definition of this gait 2. The time duration of the double support phase, that is an optimisation variable, for the gait 2 is smaller than for the gait 1 . It could be a reason why the consumption of energy is less for the gait 2 than for the gait 1 because the motion of the swing leg is quasi ballistic. ${ }^{41}$ 


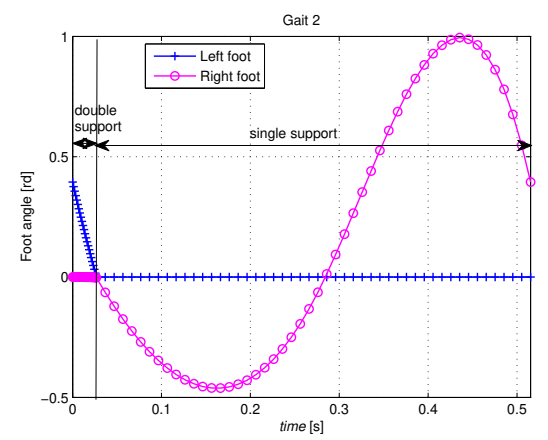

Fig. 13. Profile of the foot orientations with respect to the ground for the gait 2 .

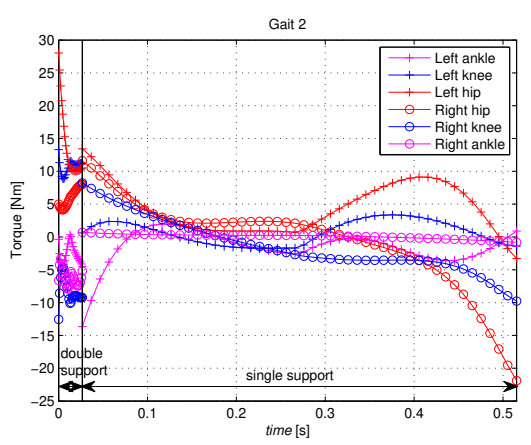

Fig. 14. Profile of the torques for the gait 2 .

\section{Conclusion}

This paper deals with the design of optimal periodic walking motions with finite time double support phases and single support phases for a planar biped through a parametric optimization. The three original main results of this numerical study are the following. Firstly, with the algebraic model of impact the fact to accept a rotation of the rear foot on its toe, which was the previous stance foot in single support, allows to get a valid non instantaneous double support phase. That means this model satisfies the unilateral constraints i.e. the vertical component of the impulsive ground reaction is positive for the two feet and the tangential force is in the friction cone. It is true for all the calculated walking motions for a landing of the swing foot on its heel or with flat foot contact. Secondly the range of allowable speeds is greater for the finite time double support phase where the swing foot is landing with a flat foot contact on the ground than for the finite double support phase that allows a synchronized rotation of both feet. For both kinds of finite time double support phases, beyond the upper and lower limits in speeds the optimization algorithm cannot find any optimal solution, which satisfies the unilateral constraints. Thirdly the gait 1 , which has a flat foot contact in finite time double support phase, is optimal for the low velocities. The gait 2, which allows a rotation of both feet, is optimal for high biped speeds. This last result is coherent with the observations of the biomechanical data from several researchers in biomechanics. ${ }^{42}$

The developed tools here could be useful in the design of a prothesis or an exoskeleton for rehabilitation, specially to tune the assistance for the locomotor system. Walking motion designed for healthy people can be defined as a reference motion to track for handicapped people by tuning the assistance of the prothesis or the exoskeleton during the gait. Preliminary results can be found in. ${ }^{43}$ 


\section{References}

1. R. Alexander, The gaits of bipedal and quadrupedal animals, International Journal of Robotics Research 3 (4) (1984) 49-59 (1984).

2. R. Singh, H. Chaudhary, A. K. Singh, Sagittal position analysis of gait cycle for a five link biped robot, Springer, 2016, pp. 387-396 (2016).

3. J. Saunders, T. I. Verne, D. E. Howard, The major determinants in normal and pathological gait, Journal Bone and Joint Surgery 35 (3) (1953) 543-558 (1953).

4. D. Winter, Biomechanics and motor control of human movement, Wiley, 2005 (2005).

5. C. Chevallereau, G. Bessonnet, G. Abba, Y. Aoustin, Bipedal Robots, ISTE Wiley, 2009 (2009).

6. S. Nadubettu Yadukumar, M. Pasupuleti, A. Ames, Human-inspired underactuated bipedal robotic walking with amber on flat-ground, up-slope and uneven terrain, in: IEEE/RSJ International Conference on Intelligent Robots and Systems, Vilamoura, Algarve, Portugal, 2012 (October 2012).

7. C. Chevallereau, G. Abba, Y. Aoustin, F. Plestan, E. Westervelt, C. Canuddas-de Wit, J. Grizzle, Rabbit: a testbed for advanced control theory, IEEE Control Systems Magazine 23 (5) (2003) 57-79 (2003).

8. S. Kajita, F. Kanehiro, M. Morisawa, S. Nakaoka, H. Hirukawa, Biped walking pattern generator allowing auxiliary ZMP control, in: 2006 IEEE/RSJ International Conference on Intelligent Robots and Systems, Beijing, P.R. China, 2006, pp. 2993-2999 (2006).

9. S. Omran, S. Sakka, Y. Aoustin, Effects of com vertical oscillation on joint torques during 3D walking of humanoid robots, Int. J. of Humanoid Robotics (DOI:10.1142/S02198436165001951650019, 2016).

10. S. Miossec, Y. Aoustin, A simplified stability for a biped walk with under and over actuated phase, International Journal of Robotics Research 24 (7) (2005) 537-551 (2005).

11. K. K. Hamed, N. Sadati, W. A. Gruver, G. A. Dumont, Stabilization of periodic orbits for planar walking with noninstantaneous double-support phase, IEEE Trans. on Systems, Man, and CyberneticsPart A: Systems and Humans 42 (3) (2012) 685-706 (2012).

12. M. S. Ju, J. M. Mansour, Simulation of the double limb support phase of human gait, Journal of Biomechanical Engineering 110 (3) (1988) 223-229 (1988).

13. N. Sharma, R. Stein, Gait planning and double support phase model for functional electrical stimulation-based walking, in: $33^{t} h$ Annual International Conference of the IEEE EMBS, San Diego, California USA, 2012 (August 2012).

14. G. Bessonnet, S. Chesse, P. Sardain, Optimal Gait Synthesis of a Seven-Link Planar Biped, The International Journal of Robotics Research 23 (10-11) (2004) 1059-1073 (2004).

15. D. Tlalolini Romero, C. Chevallereau, Y. Aoustin, Comparison of different gaits with rotation of the feet for a planar biped, Robotics and Autonomous Systems 57 (4) (2009) 371-383 (Avril 2009).

16. M. Rostami, G. Bessonnet, Impacless sagittal gait of a biped robot during the single support phase, in: IEEE International Conference on Robotics and Automation ICRA'98, Leuven, Belgium, 1998 (May 1998).

17. Y. Aoustin, A. Hamon, Human like trajectory generation for a biped robot with a four-bar linkage for the knees, Robotics and Autonomous Systems 61 (12) (2013) 1717-1725 (2013).

18. M. Tan, L. Jenning, S. Wang, Analysing human walking using dynamic optimisation, Springer, Berlin, Heidelberg, 2015, pp. 1-34 (2015).

19. D. Dadashzadeh, C. J. B. Macnab, Slip-based control of bipedal walking based on two-level control strategy, Robotica 38 (8) (2020) 1434-1449 (2020).

20. T. Kinugasa, C. Chevallereau, Y. Aoustin, Effect of circular arc feet on a control law for a biped, Robotica 27 (4) (2009) 621-632 (2009).

21. S. Bertrand, O. Bruneau, F. B. Ouezdou, S. Alfayad, Closed-form solutions of inverse kinematic models for the control of a biped robot with 8 active degrees of freedom per leg, Mechanism and machine theory, Elsevier 49 (2012) 117-140 (2012).

22. C. Chevallereau, Y. Aoustin, Optimal reference trajectories for walking and running of a biped robot, Robotica 19 (5) (2001) 557-569 (2001).

23. S. Alfayad, Robot humanoïde hydroïd: Actionnement structure cinématique et stratégie de contrôle, Ph.D. thesis, Université de Versailles Saint-Quentin (2009).

24. E. P. Hanavan, A personalized mathematical model of the human body, Journal of Spacecraft and rockets 3 (3) (1966) 446-448 (1966).

25. A. E. Bryson, Y. C. Ho, Applied optimal control, Wiley, New-York, 1995 (1995).

26. G. Vainikko, On the stability and convergence of the collocation method, Differen. Uravnen. 1 (1965) $244-254$ (1965).

27. M. K. E. Daou, E. L. Ortiz, A recursive formulation of collocation in terms of canonical polynomials, Journal of Applied Mathematics and Mechanics. 85 (2005) 177-202 (2005).

28. K. Mombaur, H. G. Bock, J. P. Schlöder, R. W. Longman, Open-loop stable solutions of periodic optimal control problems in robotics, ZAMM 85 (7) (2005) 499-515 (2005).

29. F. D. Groote, A. L. Kinney, A. V. Rao, B. J. Fregly, Evaluation of direct collocation optimal control problem formulations for solving the muscle redundancy problem, Annals of Biomedical Engineering 44 (10) (2016) 2922-2936 (2016).

30. L. Roussel, C. C. de Wit, A. Goswami, Generation of energy optimal complete gait cycles for biped, in: Proc. of the IEEE Conf. on Robotics and Automation, 1998, pp. 2036-2041 (1998). 
31. V. V. Beletskii, P. S. Chudinov, Parametric optimization in the problem of bipedal locomotion, Izv. An SSSR. Mekhanika Tverdogo Tela [Mechanics of Solids] (1) (1977) 25-35 (1977).

32. P. H. Channon, S. H. Hopkins, D. T. Pham, Derivation of optimal walking motions for a bipedal walking robot, Robotica 10 (3) (1992) 165-172 (1992).

33. G. Cabodevilla, N. Chaillet, G. Abba, Energy-minimized gait for a biped robot, in: Proceedings of the AMS'95, Tampa, Florida USA, 2002, pp. 90-99 (2002).

34. Q. Huang, K. Yokoi, S. Kajita, K. Kaneko, H. Arai, N. Koyachi, K. Tanie, Planning walking patterns for a biped robot, IEEE Trans. on Robotics and Automation 17 (3) (2001) 280-289 (2001).

35. D. Roberts, J. Quacinella, J. H. Kim, Energy expenditure of a biped walking robot: instantaneous and degree-of-freedom-based instrumentation with human gait implications, Robotica 35 (5) (2017) 1054-1071 (2017).

36. C. D. Boor, A practical guide to splines, Springer-Verlag, 1978 (1978).

37. P. Gill, W. Murray, M. Wright, Practical optimization, Academic Press, London, 1981 (1981).

38. M. Powell, Variable metric methods for constrained optimization, Lecture Notes in Mathematics, Springer Berlin / Heidelberg, 1977, pp. 62-72 (1977).

39. S. Miossec, Y. Aoustin, Dynamical synthesis of a walking cyclic gait for a biped with point feet, Vol. 340 of Lecture Notes in Control and Information Sciences, Springer Berlin / Heidelberg, 2006, pp. $233-252$ (2006).

40. J. Cottalorda, C. Durst, R. Aubail, A. Belli, V. Gautheron, Geyssant, Consommation énergétique à la vitesse de confort au sol et sur tapis roulant, Annales de Réadaptation et de Médecine Physique, Editions scientifiques et médicales Elsevier 43 (1) (2000) 30-35 (2000).

41. S. Mochon, T. McMahon, Ballistic walking: An improved model, Mathematical Bio-sciences 52 (1981) 241-260 (1981).

42. J. Rose, G. Gamble, Human Walking, Third Edition, Lippincott Williams \& Wilkins, 2006 (2006)

43. Y. Aoustin, C. Chevallereau, V. Arakelian, Study and choice of actuation for a walking assist device, Springer, 2016, pp. 3-12 (2016).

\section{Appendix}

\section{A. The equations of contact with the ground for the biped in position, velocity, and acceleration.}

Equations for legs 1 and 2 are similar with different joint variables. For a sake of clarity we consider leg 1 only.

- Contact with the heel

$$
\begin{gathered}
x+l_{2} \sin q_{2}+l_{1} \sin q_{1}-l_{f} \cos q_{f 1}+H_{f} \sin q_{f 1}=\text { const } \\
y-l_{2} \cos q_{2}-l_{1} \cos q_{1}-l_{f} \sin q_{f 1}-H_{f} \cos q_{f 1}=0 .
\end{gathered}
$$

The first time derivative of (A1) is:

$$
\begin{gathered}
\dot{x}+l_{2} \dot{q}_{2} \cos q_{2}+l_{1} \dot{q}_{1} \cos q_{1}+l_{f} \dot{q}_{f 1} \sin q_{f 1}+ \\
H_{f} \dot{q}_{f 1} \cos q_{f 1}=0, \\
\dot{y}+l_{2} \dot{q}_{2} \sin q_{2}+l_{1} \dot{q}_{1} \sin q_{1}-l_{f} \dot{q}_{f 1} \cos q_{f 1}+ \\
H_{f} \dot{q}_{f 1} \sin q_{f 1}=0 .
\end{gathered}
$$

In compact form (A2) becomes: $\mathbf{J}_{h 1} \dot{\mathbf{q}}=\mathbf{0}$ with

$$
\mathbf{J}_{h 1}=\left[\begin{array}{ccccccccc}
l_{f} \sin q_{f 1}+H_{f} \cos q_{f 1} & 0 & l_{1} \cos q_{1} & l_{2} \cos q_{2} & 0 & 0 & 0 & 1 & 0 \\
-l_{f} \cos q_{f 1}+H_{f} \sin q_{f 1} & 0 & l_{1} \sin q_{1} & l_{2} \sin q_{2} & 0 & 0 & 0 & 0 & 1
\end{array}\right] .
$$


The second time derivative of $(\mathrm{A} 1)$ is:

$$
\begin{gathered}
\ddot{x}+l_{2} \ddot{q}_{2} \cos q_{2}+l_{1} \ddot{q}_{1} \cos q_{1}+l_{f} \ddot{q}_{f 1} \sin q_{f 1}+ \\
H_{f} \ddot{q}_{f 1} \cos q_{f 1}-l_{2} \dot{q}_{2}^{2} \sin q_{2}-l_{1} \dot{q}_{1}^{2} \sin q_{1}+ \\
l_{f} \dot{q}_{f 1}^{2} \cos q_{f 1}-H_{f} \dot{q}_{f 1}^{2} \sin q_{f 1}=0, \\
\ddot{y}+l_{2} \ddot{q}_{2} \sin q_{2}+l_{1} \ddot{q}_{1} \sin q_{1}-l_{f} \ddot{q}_{f 1} \cos q_{f 1}+ \\
H_{f} \ddot{q}_{f 1} \sin q_{f 1}+l_{2} \dot{q}_{2}^{2} \cos q_{2}+l_{1} \dot{q}_{1}^{2} \cos q_{1}+ \\
l_{f} \dot{q}_{f 1}^{2} \sin q_{f 1}+H_{f} \dot{q}_{f 1}^{2} \cos q_{f 1}=0 .
\end{gathered}
$$

In compact form (A4) becomes: $\mathbf{J}_{h 1} \ddot{\mathbf{q}}+\dot{\mathbf{J}}_{h 1} \dot{\mathbf{q}}=\mathbf{0}$.

- Contact with the toe

$$
\begin{gathered}
x+l_{2} \sin q_{2}+l_{1} \sin q_{1}+\left(L_{f}-l_{f}\right) \cos q_{f 1}+H_{f} \sin q_{f 1}=\text { const }, \\
y-l_{2} \cos q_{2}-l_{1} \cos q_{1}+\left(L_{f}-l_{f}\right) \sin q_{f 1}-H_{f} \cos q_{f 1}=0 .
\end{gathered}
$$

The first time derivative of (A5) is:

$$
\begin{gathered}
\dot{x}+l_{2} \dot{q}_{2} \cos q_{2}+l_{1} \dot{q}_{1} \cos q_{1}-\left(L_{f}-l_{f}\right) \dot{q}_{f 1} \sin q_{f 1}+ \\
H_{f} \dot{q}_{f 1} \cos q_{f 1}=0, \\
\dot{y}+l_{2} \dot{q}_{2} \sin q_{2}+l_{1} \dot{q}_{1} \sin q_{1}+\left(L_{f}-l_{f}\right) \dot{q}_{f 1} \cos q_{f 1}+ \\
H_{f} \dot{q}_{f 1} \sin q_{f 1}=0 .
\end{gathered}
$$

In compact form (A6) becomes: $\mathbf{J}_{t 1} \dot{\mathbf{q}}=\mathbf{0}$ with

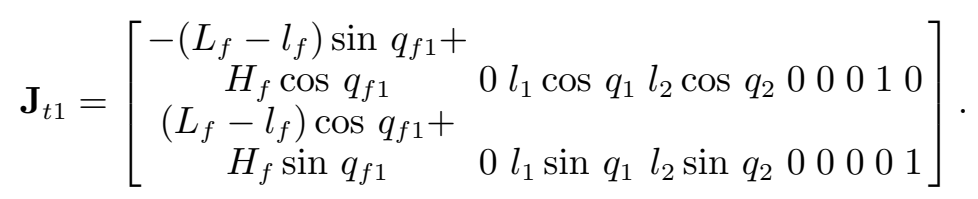

The second time derivative of (A5) is:

$$
\begin{gathered}
\ddot{x}+l_{2} \ddot{q}_{2} \cos q_{2}+l_{1} \ddot{q}_{1} \cos q_{1}-\left(L_{f}-l_{f}\right) \ddot{q}_{f 1} \sin q_{f 1}+ \\
H_{f} \ddot{q}_{f 1} \cos q_{f 1}-l_{2} \dot{q}_{2}^{2} \sin q_{2}-l_{1} \dot{q}_{1}^{2} \sin q_{1}- \\
\left(L_{f}-l_{f}\right) \dot{q}_{f 1}^{2} \cos q_{f 1}-H_{f} \dot{q}_{f 1}^{2} \sin q_{f 1}=0, \\
\ddot{y}+l_{2} \ddot{q}_{2} \sin q_{2}+l_{1} \ddot{q}_{1} \sin q_{1}+\left(L_{f}-l_{f}\right) \ddot{q}_{f 1} \cos q_{f 1}+ \\
H_{f} \ddot{q}_{f 1} \sin q_{f 1}+l_{2} \dot{q}_{2}^{2} \cos q_{2}+l_{1} \dot{q}_{1}^{2} \cos q_{1}- \\
\left(L_{f}-l_{f}\right) \dot{q}_{f 1}^{2} \sin q_{f 1}+H_{f} \dot{q}_{f 1}^{2} \cos q_{f 1}=0 .
\end{gathered}
$$

In compact form (A8) becomes: $\mathbf{J}_{t 1} \ddot{\mathbf{q}}+\dot{\mathbf{J}}_{t 1} \dot{\mathbf{q}}=\mathbf{0}$.

- Flat foot contact

$$
\begin{gathered}
x+l_{2} \sin q_{2}+l_{1} \sin q_{1}+H_{f} \sin q_{f 1}=\text { const } \\
y-l_{2} \cos q_{2}-l_{1} \cos q_{1}-H_{f} \cos q_{f 1}=0 . \\
q_{f 1}=0
\end{gathered}
$$


The first time derivative of (A9) is:

$$
\begin{gathered}
\dot{x}+l_{2} \dot{q}_{2} \cos q_{2}+l_{1} \dot{q}_{1} \cos q_{1}+H_{f} \dot{q}_{f 1} \cos q_{f 1}=0 \\
\dot{y}+l_{2} \dot{q}_{2} \sin q_{2}+l_{1} \dot{q}_{1} \sin q_{1}+H_{f} \dot{q}_{f 1} \sin q_{f 1}=0 . \\
\dot{q}_{f 1}=0
\end{gathered}
$$

In compact form (A10) becomes: $\mathbf{J}_{f 1} \dot{\mathbf{q}}=\mathbf{0}$

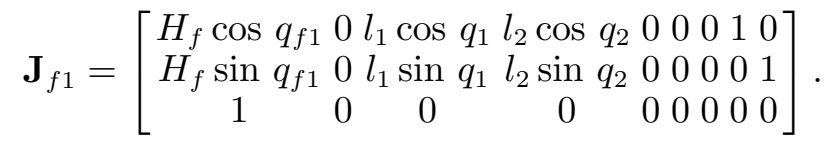

The second time derivative of (A9) is:

$$
\begin{gathered}
\ddot{x}+l_{2} \ddot{q}_{2} \cos q_{2}+l_{1} \ddot{q}_{1} \cos q_{1}+H_{f} \ddot{q}_{f 1} \cos q_{f 1}- \\
l_{2} \dot{q}_{2}^{2} \sin q_{2}-l_{1} \dot{q}_{1}^{2} \sin q_{1}-H_{f} \dot{q}_{f 1}^{2} \sin q_{f 1}=0, \\
\ddot{y}+l_{2} \ddot{q}_{2} \sin q_{2}+l_{1} \ddot{q}_{1} \sin q_{1}+H_{f} \ddot{q}_{f 1} \sin q_{f 1}+ \\
l_{2} \dot{q}_{2}^{2} \cos q_{2}+l_{1} \dot{q}_{1}^{2} \cos q_{1}+H_{f} \dot{q}_{f 1}^{2} \cos q_{f 1}=0 . \\
\ddot{q}_{f 1}=0
\end{gathered}
$$

In compact form (A12) becomes: $\mathbf{J}_{f 1} \ddot{\mathbf{q}}+\dot{\mathbf{J}}_{f 1} \dot{\mathbf{q}}=\mathbf{0}$.

\section{B. Expression of Matrix K.}

- $\mathbf{K}(1,1)=H_{f} \cos q_{f 2}-\left(L_{f}-l_{f}\right) \sin q_{f 2}+l_{2}\left(\cos q_{3}-\cos q_{2}\right)+l_{1}\left(\cos q_{4}-\cos q_{1}\right)$.

- $\mathbf{K}(2,1)=H_{f} \cos q_{f 2}-\left(L_{f}-l_{f}\right) \sin q_{f 2}+l_{2}\left(\cos q_{3}-\cos q_{2}\right)+l_{1} \cos q_{4}$.

- $\mathbf{K}(3,1)=H_{f} \cos q_{f 2}-\left(L_{f}-l_{f}\right) \sin _{f 2}+l_{2} \cos q_{3}+l_{1} \cos q_{4}$.

- $\mathbf{K}(4,1)=-H_{f} \cos q_{f 2}+\left(L_{f}-l_{f}\right) \sin q_{f 2}-l_{2} \cos q_{3}-l_{1} \cos \left(q_{4}\right.$.

- $\mathbf{K}(5,1)=-H_{f} \cos q_{f 2}+\left(L_{f}-l_{f}\right) \sin q_{f 2}-l_{1} \cos q_{3}$.

- $\mathbf{K}(6,1)=-H_{f} \cos q_{f 2}+\left(L_{f}-l_{f}\right) \sin q_{f 2}$.

- $\mathbf{K}(1,2)=H_{f} \sin q_{f 2}+\left(L_{f}-l_{f}\right) \cos q_{f 2}+l_{2}\left(\sin q_{3}-\sin q_{2}\right)+l_{1}\left(\sin q_{4}-\sin q_{1}\right)$.

- $\mathbf{K}(2,2)=H_{f} \sin q_{f 2}+\left(L_{f}-l_{f}\right) \cos q_{f 2}+l_{2}\left(\sin q_{3}-\sin q_{2}\right)+l_{1} \sin q_{4}$.

- $\mathbf{K}(3,2)=H_{f} \sin q_{f 2}+\left(L_{f}-l_{f}\right) \cos q_{f 2}+l_{2} \sin q_{3}+l_{1} \sin q_{4}$.

- $\mathbf{K}(4,2)=-H_{f} \sin q_{f 2}-\left(L_{f}-l_{f}\right) \cos f_{2}-l_{2} \sin q_{3}-l_{1} \sin q_{4}$.

- $\mathbf{K}(5,2)=-H_{f} \sin q_{f 2}-\left(L_{f}-l_{f}\right) \cos q_{f_{2}}-l_{1} \sin q_{4}$.

- $\mathbf{K}(6,2)=-H_{f} \sin q_{f 2}-\left(L_{f}-l_{f}\right) \cos q_{f 2}$. 